\title{
Hypoxia-induced internalization of connexin 26 and connexin 43 in pulmonary epithelial cells is involved in the occurrence of non-small cell lung cancer via the P53/MDM2 signaling pathway
}

\author{
SHANG-GAN ZENG ${ }^{1}$, XIANG LIN ${ }^{1}$, JI-CHUN LIU ${ }^{2}$ and JING ZHOU ${ }^{3}$ \\ ${ }^{1}$ Department of Thoracic Surgery, The First Affiliated Hospital of Nanchang University; \\ ${ }^{2}$ Departments of Cardio-Thoracic Surgery, The Second Affiliated Hospital of Nanchang University; \\ ${ }^{3}$ Department of Respiratory, The First Affiliated Hospital of Nanchang University, Nanchang, Jiangxi 330006, P.R. China
}

Received July 16, 2018; Accepted April 16, 2019

DOI: 10.3892/ijo.2019.4867

\begin{abstract}
Reports have highlighted an association between connexins (CXs) or gap junction proteins and non-small cell lung cancer (NSCLC). In the present study, it was aimed to elucidate the regulatory mechanism of CX26 and CX43 under hypoxic conditions in NSCLC. Clinical samples were collected for analysis of CX26 and CX43 expression and clinical cancerization followed by quantification of CX26 and CX43 expression. Following the establishment of an in vitro hypoxia model, $\mathrm{P} 53$ /murine double minute-2 (MDM2) signaling pathway-, proliferation- and epithelial-mesenchymal transition (EMT)-related genes were quantified to evaluate the influence of CX26 and CX43 on the biological functions of pulmonary epithelial cells in NSCLC. In addition, the proliferation and tumorigenicity of cancer cells were assessed by EdU staining and xenograft tumors, respectively. Decreased expression of CX26 and CX43 was found in cancer tissues compared with surrounding normal tissue. Hypoxia was shown to activate the P53/MDM2 axis and stimulate the downregulation, ubiquitination and degradation of CX26 and CX43, which were translocated from the membrane to the cytoplasm. Low levels of CX26 and CX43 were demonstrated to further promote EMT and the induction of the proliferation and tumorigenicity of cancer cells. These results were reflected by decreased E-cadherin expression and increased $\mathrm{N}$-cadherin expression, along with increased cell migration, promoted cell proliferation ability and elevated relative protein expression of Oct4 and Nanog, and accelerated tumor growth, accompanied by a higher number of metastatic nodes. Taken together, the
\end{abstract}

Correspondence to: Dr Jing Zhou, Department of Respiratory, The First Affiliated Hospital of Nanchang University, 17 Yongwaizheng Street, Donghu, Nanchang, Jiangxi 330006, P.R. China E-mail: zhoujindr@163.com

Key words: connexin 26, connexin 43, non-small cell lung cancer, pulmonary epithelial cells, P53/murine double minute-2 signaling pathway, proliferation, migration, epithelial-mesenchymal transition key observations of the present study demonstrate that the internalization of CX26 and CX43 promoted proliferation, EMT and migration and thus induced NSCLC via aberrant activation of the P53/MDM2 signaling pathway under hypoxic conditions.

\section{Introduction}

Lung cancer is widely acknowledged to be among the most severe and most common malignancies worldwide, with $\sim 228,150$ newly diagnosed cases and 142,670 estimated deaths in the United States in 2019 (1). Non-small cell lung cancer (NSCLC) accounts for $\sim 85 \%$ of all lung cancer cases and is the predominant form of the disease largely attributed to cancer-related deaths. Fortuitously, more effective treatment protocols have been developed in recent years that have aided in the ultimate goal of cancer remission, including improved screening methods, surgical procedures, chemoradiotherapy and diagnostic evaluation (2). However, despite the substantial progress in the last few years, the majority of NSCLC prognoses remain poor, with a $10 \%$ 5-year survival rate (3). Additionally, patients with NSCLC have been widely documented to experience poor sleep quality and unexpected sleep disturbances (4).

Obstructive sleep apnea-hypopnea (OSAH) syndrome is a condition characterized by various respiratory events during a period of sleep accompanied by loud snoring, excessive daytime sleepiness or breathing pauses (5). The morbidity of this syndrome is $3-7 \%$ among males and 2-5\% among females, and is as high as $33 \%$ in patients from Sao Paulo with low socio-economic status, with a apnea-hypopnea index between 5 to 15 ; however, research into this condition has been inadequate in recent years (6). Thus, the current study sought to identify a novel therapeutic approach in regard to the involvement of OSAH model in NSCLC.

Connexin (CX) 26 is a transmembrane protein that is part of gap junctions between adjacent cells and its connection with the PI3K/Akt signaling pathway leads to the acquired gefitinib resistance in NSCLC cells through the activation of epithelial-mesenchymal transition (EMT) (7). More specifically, CX26 has been reported to contribute to the proliferation 
of epithelial cells involved in basal airway repair (8). The CX-CX interaction mechanism has been revealed to be important in intercellular communication regulation; for instance, CX26 can be co-expressed with CX43 in human skin (9). Researchers have highlighted the role of CX26 and CX43 signaling in the suppression of tumor progression (10). In recent years, the interaction between the tumor suppressor gene P53 and its negative regulator, murine double minute-2 (MDM2), has been reported as a potential therapeutic target for patients with cancer (11). P53 has been demonstrated to induce apoptosis through blockade of cellular DNA damage repair, while the transactivation ability and stability of P53 can be repressed by MDM2 through ubiquitination (12). Based on the aforementioned literature, the present study subsequently asserted the hypothesis that an OSAH model may induce the internalization of CX26 and CX43 in pulmonary epithelial cells (PECs) with the involvement of the P53/MDM2 signaling pathway.

\section{Materials and methods}

Ethics statements. The study was approved by the Ethics Committee of The First Affiliated Hospital of Nanchang University. Written informed consent was obtained from each participant. All animal experimentation in this study was approved by the Institutional Animal Care and Use Committee of The First Affiliated Hospital of Nanchang University.

Study subjects. In total, 120 specimens (cancer pulmonary epithelial tissues and paracancerous tissues) were obtained from 60 patients with pathologically confirmed NSCLC that had previously undergone surgical resection in The First Affiliated Hospital of Nanchang University between January 2010 and December 2015. There were 38 males and 22 females with a mean age of $62.4 \pm 5.9$ years. None of the patients had OSAH. Each patient was free of any immunologically-mediated disease, connective tissue disease or comorbidity of significant organs, and did not receive anticancer therapy, including chemoradiotherapy or immunotherapy prior to surgery. The paracancerous tissues were confirmed to lack any pathologic features of cancerization according to hematoxylin-eosin (HE) staining.

Cell model establishment with hypoxia. Following isolation from one patient and culture (13), PECs derived from human NSCLC were washed twice with PBS and cultured in DMEM/F12 (cat. no. GNM-12500-S; Shanghai Jingke Chemical Technology Co., Ltd.) containing 20\% FBS (Sigma-Aldrich; Merck KGaA) in an incubator with $5 \% \mathrm{CO}_{2}$ at $37^{\circ} \mathrm{C}$. The medium was changed once every 3 days as soon as the majority of the epithelial cells were detached. In the event that cell confluence reached $80-90 \%$, the medium was subsequently discarded. The cells were then washed twice with PBS and detached following treatment with $0.25 \%$ trypsin, after which the density was adjusted to $1 \times 10^{6}$ cells $/ \mathrm{ml}$ using DMEM containing $10 \%$ calf serum (Sigma-Aldrich; Merck KGaA). The cells were then further cultured in the plate and dish.

An in vitro model was established by exposing the cells to hypoxic conditions over an extended period. The cells were assigned to two groups: Hypoxia and normal group.
Intermittent hypoxia under normal pressure and temperature was applied in in vitro model, which was established to mimic the conditions of chronic intermittent hypoxia that occurs in the body due to snoring during the night. A transparent plexiglass box was used ( $5 \mathrm{~mm}$ in thickness; $75 \times 50 \times 50 \mathrm{~cm})$ in which nitrogen $(>95.5 \%)$ was input circularly $(\sim 8 \mathrm{~min} / \mathrm{cycle})$ by the experimenter. The oxygen concentration in the intermittent hypoxia box was monitored using an oxygen monitor to control the amount of air-input and air-removal. The minimum oxygen concentration required was $8.5 \%$ during each cycle. With the removal of hypoxic gas and the input of air (oxygen concentration, 20.9\%), the oxygen concentration was gradually restored to $21 \%$. The volume fraction of oxygen in the gas mixture was adjusted on a timely basis to $10 \pm 1.5 \%$ under the dynamic monitoring of the gas analyzer (OX-100A; Jiande Meicheng Analysis Instrument Factory). The water and $\mathrm{CO}_{2}$ content in the intermittent hypoxia box were absorbed using anhydrous calcium chloride and $\mathrm{CO}_{2}$ absorbent, both of which, along with water and padding in the hypoxia box, were changed every 2 days. Cells in the normal group were cultured under normal pressure and temperature with $\mathrm{O}_{2}$ (volume fraction, $21 \%$ ) between 10:00 p.m. and 06:00 a.m. each day in the dark. Cells in the hypoxia group were cultured under intermittent hypoxia with $\mathrm{O}_{2}$ (volume fraction, 8.5\%) between 10:00 p.m. and 06:00 a.m. each day in the dark (14-16).

Cell treatment. All plasmids used for transfection were purchased from Sangon Biotech Co., Ltd. The siRNA was expressed from plasmid vectors. Hsp70 siRNA expression vector was constructed by psuper-puro expression system (a primitive siRNA design) and overexpression vector was constructed using pcDNA3.1-GALT-GFP. Cells in the exponential growth phase were seeded into a 24 -well plate at $500 \mu 1 /$ well with a final cell density adjusted to $1.2 \times 10^{5}$ cells $/ \mathrm{ml}$. The transfection was performed in accordance with the instructions of the Lipofectamine $^{\circledR} 2000$ kit (cat. no. 11668-019; Invitrogen; Thermo Fisher Scientific, Inc.). Briefly, 100 pmol si-CX26, si-CX43, CX26, CX43 and negative control (NC), or target plasmid were diluted in $250 \mu$ l serum-free Opti-MEM (Gibco; Thermo Fisher Scientific, Inc.; final concentration of $50 \mathrm{nM}$ in the cells), mixed and incubated at room temperature for $5 \mathrm{~min}$; $4 \mu \mathrm{g}$ was used for plasmids. An additional $250 \mu \mathrm{l}$ serum-free Opti-MEM was used to dilute $5 \mu 1$ Lipofectamine 2000 followed by a 5 -min incubation at room temperature. The two solutions were then mixed, incubated at room temperature for $20 \mathrm{~min}$ and added to the wells. Following incubation at $37^{\circ} \mathrm{C}$ with $\mathrm{CO}_{2}$ for 6-8 h, the cells were further cultured in complete culture medium for $48 \mathrm{~h}$ for the following experiments. The sequences of the siRNAs were as follows: si-CX26, 5'-CCC AGUUGUUAGAUUAAGATT-3'; si-CX26-NC, 5'-UUCUCC GAACGUGUCACGUTT-3'; si-CX43, 5'-AGAGAUACGAAC AAGAGAG-3'; si-CX43-NC, 5'-AUACAACAGAGAGGG AAAG-3'.

The cells were assigned to 14 groups, as follows: Normal (PECs exposed to normal oxygen); hypoxia (PECs exposed to hypoxia); hypoxia + lactacystin (6 $\mu \mathrm{M}$ proteasome inhibitors) (17); normal + lactacystin $(6 \mu \mathrm{M}$ proteasome inhibitors); si-NC-CX26 + si-NC-CX43 (PECs transfected with NC), si-CX26 + si-NC-CX43 (PECs transfected with si-CX26 and NC of si-CX43); 
si-NC-CX26 + si-CX43 (PECs transfected with si-CX43 and $\mathrm{NC}$ of si-CX26); si-CX26 + si-CX43 (PECs transfected with si-CX26 and si-CX43); NC-CX26 + NC-CX43 + hypoxia; CX26 + NC-CX43 + hypoxia; NC-CX26 + CX43 + hypoxia; CX26 + CX43 + hypoxia; hypoxia + monodansylcadaverine (MDC; $200 \mu \mathrm{mol} / 1$ protein internalization inhibitors) (18); and normal + MDC. A flow-chart (Fig. S1) was created to demonstrate the experimental design.

Immunohistochemistry for CX26 and CX43 expression. The specimens were fixed in $10 \%$ formaldehyde at room temperature for $5 \mathrm{~min}$, embedded in paraffin and continuously sliced at a thickness of $4 \mu \mathrm{m}$. The tissue sections were heated in an oven at $60^{\circ} \mathrm{C}$ for $1 \mathrm{~h}$, followed by conventional xylene deparaffinization and dehydration in gradient alcohol (100, 95 and $80 \%)$ at room temperature for 10-15 min each. The slices were then incubated in $3 \% \mathrm{H}_{2} \mathrm{O}_{2}$ (Sigma-Aldrich; Merck KGaA) at $37^{\circ} \mathrm{C}$ for $30 \mathrm{~min}$, rinsed with PBS and boiled in $0.01 \mathrm{M}$ citrate buffer at $95^{\circ} \mathrm{C}$ for $20 \mathrm{~min}$. After they had cooled to room temperature, the slices were washed with PBS and then blocked with $10 \%$ normal goat serum (Sigma-Aldrich; Merck KGaA) at $37^{\circ} \mathrm{C}$ for $10 \mathrm{~min}$. Subsequently, the slices were incubated with the following primary antibodies overnight at $4^{\circ} \mathrm{C}: 50 \mu 1$ rabbit anti-mouse CX26 (1:100; cat. no. ab65969; Abcam) and CX43 (1:1,000; cat. no. ab11370; Abcam). After they were rinsed in PBS, the sections were incubated with the goat anti-rabbit IgG secondary antibody (cat. no. ab150077; 1:1,000; Abcam) for $30 \mathrm{~min}$ at $37^{\circ} \mathrm{C}$, which was followed by three washes in PBS ( 3 min each time). Next, the slices were developed using 3,3'-diaminobenzidine (Sigma-Aldrich; Merck KGaA) at room temperature for $5 \mathrm{~min}$, counterstained in 0.5\% hematoxylin (Shanghai Bogoo Biotech Co., Ltd.) at room temperature for $3 \mathrm{~min}$ and sealed. Slides for which PBS was used instead of the primary antibody served as negative controls, while normal tissue served as the positive control. The samples were observed under an optical microscope (XSP-36, Shenzhen Boshida Optical Instrument Co., Ltd.) and images were obtained. Five high-power fields (x200) were randomly selected from each slice, and 100 PECs were counted in each field. Fields with $<10 \%$ positive cells were considered negative, while those with $\geq 10 \%$ positive cells were deemed positive (19).

Immunofluorescence for cellular localization of CX26 and CX43. Following conventional detachment and transfection, the cells were counted and cultured in immunofluorescence chambers at a density of $2 \times 10^{5}$ cells/well. When cell confluence reached $\sim 90 \%$, the cells were washed three times with PBS on ice and fixed in $1 \mathrm{ml} 4 \%$ paraformaldehyde at room temperature for $15 \mathrm{~min}$, followed by an additional three washes in PBS. The cells were then treated with $0.3 \%$ Triton for $10 \mathrm{~min}$ at $37^{\circ} \mathrm{C}$, followed by another three washes in PBS and blocking in goat serum (Sigma-Aldrich; Merck KGaA) for $30 \mathrm{~min}$ at $37^{\circ} \mathrm{C}$. Next, the primary antibodies (prepared in PBS) against CX26 (cat. no. 33-5800; 1:400; Thermo Fisher Scientific, Inc.) and CX43 (cat. no. ab11370; 1:1,000; Abcam) were added and incubated with the cells overnight at $4^{\circ} \mathrm{C}$. After three washes in PBS, the secondary antibody to Alexa Fluor 488 (cat. no. A11029; 1:500; Invitrogen; Thermo Fisher Scientific, Inc.) was added and incubated with the sample at room temperature for $1 \mathrm{~h}$ in the dark. The cells were then washed three times with PBS, stained with DAPI staining solution for $15 \mathrm{~min}$ in the dark and washed an additional three times with PBS. A fluorescence-quenching agent was added for sealing. The samples were then finally observed and images under a fluorescence microscope.

Co-immunoprecipitation (Co-IP) for the binding of CX26 and CX43 to ubiquitin protein. The cells were washed twice with pre-cooled PBS followed by the removal of PBS. After the addition of pre-cooled RIPA buffer, the cells were scraped with a cell scraper. The suspension was subsequently transferred to a $1.5 \mathrm{ml}$ Eppendorf tube and continuously shaken for $15 \mathrm{~min}$ in a gentle manner at $4^{\circ} \mathrm{C}$, which was followed by a centrifugation at $14,000 \mathrm{x}$ g at $4^{\circ} \mathrm{C}$ for $15 \mathrm{~min}$. The supernatant was then transferred to a new centrifuge tube. Ubiquitin agarose beads (Thermo Fisher Scientific, Inc.) were prepared and washed twice with PBS. The concentration was then adjusted to 50\% using PBS. For every $1 \mathrm{ml}$ total protein, $100 \mu \mathrm{l} 50 \%$ ubiquitin agarose beads were added, which were then shaken for $10 \mathrm{~min}$ at $4^{\circ} \mathrm{C}$ to remove non-specific hybrid protein and to reduce background signal. Following centrifugation at $14,000 \mathrm{x} \mathrm{g}$ at $4^{\circ} \mathrm{C}$ for $15 \mathrm{~min}$, the supernatant was then transferred to a new centrifuge tube, and the ubiquitin agarose beads were removed. Total protein was diluted 10 -fold. A protein standard curve was plotted using the Bradford method followed by determination of the protein concentration. A total of $200 \mu 1$ diluted total protein $(10 \mu \mathrm{g} / \mu \mathrm{l})$ was added to $0.2 \mu \mathrm{l}$ primary antibody against ubiquitin (cat. no. ab19247; Abcam) and shaken gently for $1 \mathrm{~h}$ at $4^{\circ} \mathrm{C}$. Subsequently, $20 \mu \mathrm{l}$ ubiquitin agarose beads were added for incubation at $4^{\circ} \mathrm{C}$ overnight. Centrifugation was then performed at 1,500 $\mathrm{x}$ g for $5 \mathrm{~min}$ followed by the collection of the agarose beads-antigen-antibody complex and the removal of the supernatant. The complex was then washed three times with $800 \mu \mathrm{l}$ pre-cooled PBS, suspended in $60 \mu \mathrm{l} 2 \mathrm{X}$ loading buffer, gently mixed together and boiled for $5 \mathrm{~min}$. Then, the expression of CX26 and CX43 was analyzed by western blot analysis.

Reverse transcription-quantitative PCR (RT-qPCR) for determination of the expression of CX26,CX43 and downstream target genes of the P53/MDM2 signaling pathway. RT-qPCR was applied to determine the expression of CX26, CX43 and downstream target genes of the P53/MDM2 signaling pathway. The primers of the downstream target genes were designed using Primer 5.0 software (Premier Biosoft International) and were analyzed for homology using BLAST software (blast.ncbi.nlm.nih.gov/Blast.cgi) with the related reference sequences provided by GenBank (ncbi.nlm.nih.gov/genbank/), as depicted in Table I. Following transfection, the cells were collected, from which total RNA was extracted using a TRIzol (Invitrogen; Thermo Fisher Scientific, Inc.). Next, cDNA was obtained through RT in accordance with the instructions of RevertAid First Strand cDNA Synthesis Kit (cat. no. K1622; Thermo Fisher Scientific, Inc.), which was followed by PCR amplification using SYBR ${ }^{\circledR}$ Premix Ex TaqTM II (Perfect Real Time) kit (Takara Bio, Inc.). The reaction conditions were as follows: Pre-denaturation at $95^{\circ} \mathrm{C}$ for $5 \mathrm{~min}, 45$ cycles of denaturation at $95^{\circ} \mathrm{C}$ for $20 \mathrm{sec}$, annealing at $60^{\circ} \mathrm{C}$ for $1 \mathrm{~min}$ and extension at $72^{\circ} \mathrm{C}$ for $30 \mathrm{sec}$. The qPCR results were 
Table I. Primers for reverse transcription-quantitative PCR.

Name Primer sequence $\left(5^{\prime}-3^{\prime}\right)$

$\beta$-actin Forward: CATGTACGTTGCTATCCAGGC Reserve: CTCCTTAATGTCACGCACGAT

E-cadherin Forward: CGAGAGCTACACGTTCACGG Reserve: GGGTGTCGAGGGAAAAATAGG

N-cadherin Forward: TCAGGCGTCTGTAGAGGCTT Reserve: ATGCACATCCTTCGATAAGACTG

CX26 Forward: TCGCATTATGATCCTCGTTGTG Reserve: GGGGAAGTAGTGATCGTAGCAC

CX43 Forward: GGTGACTGGAGCGCCTTAG Reserve: GCGCACATGAGAGATTGGGA

P53 Forward: CAGCACATGACGGAGGTTGT Reserve: TCATCCAAATACTCCACACGC

P21 Forward: TGTCCGTCAGAACCCATGC Reserve: AAAGTCGAAGTTCCATCGCTC MDM2 Forward:GAATCATCGGACTCAGGTACATC Reserve: TCTGTCTCACTAATTGCTCTCCT

CX, connexin; MDM2, mouse double minute 2.

subsequently analyzed using the $2^{-\Delta \Delta \mathrm{Cq}}$ method (20) to determine the relative expression of the target genes at the transcriptional level. $\beta$-actin was regarded as the internal reference for CX26, CX43, $\beta$-catenin, E-cadherin, Vimentin, P53, P21 and MDM2. The experiment was repeated three times.

Western blot analysis for protein expression measurement. The cells in each group were collected, and the total protein was extracted. The concentration was determined using a BCA kit (cat. no. 20201ES76; Yeasen Biotechnology Co., Ltd.). The amount of protein was adjusted to $20 \mu \mathrm{g}$ per well. Following SDS-PAGE on $8 \%$ gels for $1 \mathrm{~h}$, the separated protein band was transferred to a PVDF membrane. The membrane was then blocked with $5 \%$ skim milk powder at room temperature for $1 \mathrm{~h}$ and incubated with rabbit polyclonal antibodies, including ubiquitin (cat. no. ab140601; 1:500), $\beta$-actin (cat. no. ab8227; 1:1,000), CX26 (cat.no.ab65969; 1:1,000), CX43 (cat.no.ab11370; 1:8,000), P53 (ab131442; 1:500), P21 (ab109199; 1:1,000), MDM2 (cat. no. ab38618; 1:1,000), E-cadherin (cat. no. ab76055; 1:1,000), Vimentin (cat. no. ab8978; 1:1,000), Oct4 (cat. no. ab181557; 1:1,000) and Nanog (cat. no. ab218524; 1:1,000) overnight at $4^{\circ} \mathrm{C}$. After three washes in PBS with Tween-20 (PBST), the membrane was incubated with the secondary antibody, horseradish peroxidase-labeled goat anti-rabbit IgG (cat. no. ab150077; 1:1,000) at room temperature for $1 \mathrm{~h}$, followed by an additional three washes in PBST. All were purchased from Abcam. The membrane was then immersed in the enhanced chemiluminescence detection solution (cat. no. ECL808-25; Biomiga, Inc.) in the dark. The images were visualized using a SmartView Pro 2000 (UVCI-2100; Major Science). The relative protein levels were expressed by the gray value of the target protein band to that of the $\beta$-actin protein band using the Quantity One v4.6.2 software (Bio-Rad Laboratories, Inc.).
Transwell assay. The cells in the logarithmic growth phase were starved for $24 \mathrm{~h}$, detached, centrifuged, and resuspended to adjust the final concentration into $2 \times 10^{5}$ cells $/ \mathrm{ml}$. The extracellular matrix (ECM) gel was allowed to stand at $4^{\circ} \mathrm{C}$ overnight and diluted with serum-free medium at a ratio of 1:9 to reach a final concentration of $1 \mathrm{mg} / \mathrm{ml}$. The diluted ECM gel $(40 \mu \mathrm{l})$ was added to the polycarbonate membrane of each 24-well Transwell apical chamber and incubated in a $5 \% \mathrm{CO}_{2}$ incubator at $37^{\circ} \mathrm{C}$ for $5 \mathrm{~h}$. After the ECM gel was polymerized to form a gel, the DMEM was added (70 $\mu 1$ each chamber). After another incubation in a $37^{\circ} \mathrm{C}$ incubator for $0.5 \mathrm{~h}$, the ECM gel was rehydrated. The cells in each group were starved for $24 \mathrm{~h}$, detached, centrifuged and resuspended with FBS-free DMEM to adjust the final concentration into $2.5 \times 10^{5}$ cells $/ \mathrm{ml}$. A total of $0.2 \mathrm{ml}$ suspension was added into the apical chamber in which the basement membrane has been hydrated, while $700 \mu 1$ pre-chilled DMEM containing 10\% FBS was supplemented into the basolateral chamber. The Transwell chamber was then cultured in a $5 \% \mathrm{CO}_{2}$ incubator at $37^{\circ} \mathrm{C}$ for $24 \mathrm{~h}$. The cells in the chamber and basement membrane were removed by wet cotton swabs, fixed with methanol for $30 \mathrm{~min}$, stained with $0.1 \%$ crystal violet for $20 \mathrm{~min}$, dried, observed under an inverted microscope and photographed. Five visual fields were randomly selected to count the number of cells crossing the membrane to take the average.

Colony formation assay. Agarose (1.2\%) was dissolved, and water-bathed in $46^{\circ} \mathrm{C}$. Counted single cells were suspended in $40^{\circ} \mathrm{C}$ pre-warmed RPMI-1640 medium containing $40 \%$ fetal calf serum. Afterwards, $325 \mu \mathrm{l}$ cell suspension $\left(1 \times 10^{5}\right.$ cells) was added into each well of the 24-well plate. Following the addition of $50 \mu \mathrm{l}$ sample to be tested, $125 \mu \mathrm{l}$ pre-warmed $1.2 \%$ agarose was supplemented. After natural coagulation, the sample was cultured in a $5 \% \mathrm{CO}_{2}$ incubator at $37^{\circ} \mathrm{C}$ for $8-10$ day and observed under an inverted microscope.

5-Ethynyl-2'-deoxyuridine (EdU) staining for cell proliferation. The cells in each group were cultured in $50 \mu \mathrm{M}$ EdU (EdU labeling/detection kit; Guangzhou RiboBio Co., Ltd.) for $12 \mathrm{~h}$, fixed in $4 \%$ paraformaldehyde for $30 \mathrm{~min}$ at room temperature and further incubated in 5\% glycine for $5 \mathrm{~min}$ at room temperature. The cells were washed in PBS followed by treatment with $0.5 \%$ Triton $\mathrm{X}-100$ at room temperature for $30 \mathrm{~min}$. The samples in each well were incubated with $100 \mu \mathrm{l}$ Apollo ${ }^{\circledR}$ mixture (cat. no. C10338-2; Guangzhou RiboBio Co., Ltd.) for $30 \mathrm{~min}$ at room temperature. The cells were observed and imaged under a fluorescence microscope.

Flow cytometry for cell cycle and cell apoptosis. At $48 \mathrm{~h}$ after transfection, the cells were collected for cell cycle analysis. The cells were resuspended in PBS $\left(\sim 1 \times 10^{6}\right.$ cells $)$, fixed in $75 \%$ ethanol at $4^{\circ} \mathrm{C}$ for $1 \mathrm{~h}$ and incubated with $150 \mu$ l RNase (cat. no. GE101-01; TransGen Biotech Co., Ltd.) for $40 \mathrm{~min}$ at $37^{\circ} \mathrm{C}$. Afterwards, the cells were stained with $200 \mu \mathrm{l}$ propidium iodide (PI; Sigma-Aldrich; Merck KGaA) for $2 \mathrm{~h}$ at $4^{\circ} \mathrm{C}$. Finally, the cell cycle distribution was measured through the detection of red fluorescence at an excitation wavelength of $488 \mathrm{~nm}$ using a flow cytometer (CytoFLEX; Beckman Coulter) and analyzed using ModfitLT for Mac 3.0 software (Verity Software House, Inc.). 
After transfection for $48 \mathrm{~h}$, the cells were treated with EDTA-free trypsin (cat. no. YB15050057; Shanghai Yu Bo Biological Technology Co., Ltd.) and collected into a flow tube. The supernatant was discarded after centrifugation at room temperature at 1,200 $\mathrm{x}$ g for $5 \mathrm{~min}$, which was followed by three washes in pre-cooled PBS. Centrifugation was then performed again after removal of the supernatant. Based on the instructions of the Annexin-V-FITC cell apoptosis detection kit (cat. no. APOAF-20TST; Sigma-Aldrich; Merck $\mathrm{KGaA}$ ), Annexin-V-FITC/PI staining solution was prepared using Annexin-V-FITC, PI and HEPES buffer at a ratio of 1:2:50. Then, $100 \mu 1$ staining solution was used to resuspend $1 \times 10^{6}$ cells, followed by oscillation and mixing. The specimens were then incubated at room temperature for $15 \mathrm{~min}$, after which $1 \mathrm{ml}$ HEPES buffer solution (cat. no. PB180325; Procell Life Science Co., Ltd.) was added and the suspension was oscillated and mixed accordingly. Cell apoptosis was analyzed through the detection of FITC and PI fluorescence by activating the band pass at 525 and $620 \mathrm{~nm}$ at a wavelength of $488 \mathrm{~nm}$ using a flow cytometer.

Xenograft tumors in nude mice. Male nude mice $(\mathrm{n}=20$; $18-22 \mathrm{~g} ; 4-6$ weeks) were housed in a temperature- $\left(26-28^{\circ} \mathrm{C}\right)$ and humidity-controlled (40-60\%) environment with a 10/14 h light/dark cycle, and were provided free access to aseptic water and chow. The cage was ventilated 10-15 times per $h$ under positive air pressure of $0.65 \mathrm{~cm} \mathrm{H}_{2} \mathrm{O}$. The animals were assigned to four groups, as follows: si-NC-CX26 + si-NC-CX43; si-CX26 + si-NC-CX43; si-NC-CX26 + si-CX43; and si-CX26 + si-CX43. After the preparation of lentivirus (2x10 $10^{7}$ TU; Shanghai GenePharma Pharmaceutical Technology Co., Ltd.) carrying siRNAs, the lentivirus-packed si-NC-CX26 + si-NC-CX43, si-CX26 + si-NC-CX43, si-NC-CX26 + si-CX43, and si-CX26 + si-CX43 were transduced into isolated human NSCLC PECs. The $2 \times 10^{6}$ cells (dissolved in $200 \mu \mathrm{l}$ normal saline) were subcutaneously injected into the right axilla of each nude mouse. From the 7 th day after injection, observations were conducted once every 7 days. The length and width of the tumors that appeared in the mice were recorded in detail. The approximate tumor volume was calculated according to the formula, volume $\left(\mathrm{mm}^{3}\right)=$ length $\mathrm{x}$ width ${ }^{2} / 2$. The mice were subsequently euthanized on the 35 th day to collect their tumors.

$H E$ staining. The lung tissues were obtained under a dissecting microscope (Olympus Corporation) $(21,22)$. The tissues were fixed in $3 \%$ neutral formalin at room temperature for $5 \mathrm{~min}$ and used to generate paraffin slices at a thickness of $5-8 \mu \mathrm{m}$. The prepared sections were deparaffinized twice in xylene (5 min each time) and dehydrated in gradient ethanol solutions $(100,95,80$ and $75 \%)$ for 1 min each. After they were washed in running water for $2 \mathrm{~min}$, the sections were stained with hematoxylin for $2 \mathrm{~min}$ and washed under running water for $10 \mathrm{sec}$. Next, the excess staining solution was removed with $1 \%$ hydrochloric acid-75\% ethanol. The sections were then washed with distilled water for $1 \mathrm{~min}$, stained with eosin for $1 \mathrm{~min}$, washed again with distilled water for $10 \mathrm{sec}$, dehydrated twice in gradient ethanol solutions (95 and 100\%; 1 min each) and cleared with xylene. All above mentioned procedures were conducted at room temperature. Finally, the sections were sealed with neutral gum for further observation.

Statistical analysis. All experimental data were processed using SPSS 19.0 statistical software (IBM Corp.). Continuous data with normal distribution and equal variance were expressed as the mean \pm standard deviation. Paired t-test was used to compare data between cancer tissues and paracancerous tissues and an independent sample t-test was used to analyze data between two groups. One-way ANOVA or two-way ANOVA (tumor volume) with Tukey's post hoc test was used for comparisons among multiple groups. Enumeration data were presented as percentages and analyzed using Fisher's exact test. $\mathrm{P}<0.05$ was considered to indicate a statistically significant difference.

\section{Results}

Hypoxia downregulates CX26 and CX43 expression in NSCLC PECs. Initially, to observe the effect of hypoxia on the expression of CX26 and CX43 in PECs derived from NSCLC, the expression of CX26 and CX43 was assessed by immunohistochemistry and RT-qPCR. As illustrated in Table II and Fig. 1A, the percentage of CX26-positive samples was $93.33 \%(56 / 60)$ in paracancerous tissues and $35.00 \%$ $(21 / 60)$ in cancer tissues $(\mathrm{P}<0.05)$. Additionally, there was a significant difference in the percentage of CX43-positive samples, which was $98.34 \%(59 / 60)$ in paracancerous tissues and $46.67 \%(28 / 60)$ in cancer tissues. The non-specific response of CX26 in the tissue background was weak, while the localization was clear with positive CX26 in a hairline or particle shape. Among paracancerous tissues, CX26-positive particles were detected on the clear-expressed membrane in a hairline shape with unstained cytoplasm, while in cancer tissues, positively stained particles were mainly distributed in the cytoplasm, and only two cases with simultaneous partly stained membranes were observed. Similar results were observed for CX43, but three cases with partly stained membranes were observed (Fig. 1A). RT-qPCR and western blot analysis revealed that CX26 and CX43 expression was decreased in cancer tissues compared with paracancerous tissues $(\mathrm{P}<0.05$; Fig. 1B and $\mathrm{C})$. Following isolation and culture, human NSCLC PECs were exposed to hypoxia, and the results revealed that CX26 and CX43 expression was significantly reduced in the hypoxia group compared with the normal group $(\mathrm{P}<0.05$; Fig. $1 \mathrm{D}$ and $\mathrm{E})$. The aforementioned data demonstrated that the expression of CX26 and CX43 was downregulated by hypoxia in PECs.

Hypoxia induces internalization and degradation of CX26 and CX43. To determine the effect of hypoxia on the internalization and degradation of CX26 and CX43, human NSCLC PECs were isolated, cultured and exposed to hypoxic conditions for extended periods followed by immunofluorescence staining. The results revealed that CX26 and CX43 were both expressed on the membrane in the normal group, while the expression and localization of CX26 were not significantly different over time under normal conditions. Compared with the normal group, the expression of CX26 and CX43 was increased in the cytoplasm, but reduced in the membranes, in the hypoxia 
Table II. Association analysis between CX26/CX43 and carcinogenesis in pulmonary epithelial tissue.

\begin{tabular}{|c|c|c|c|c|c|c|}
\hline \multirow[b]{2}{*}{ Group } & \multicolumn{3}{|c|}{ CX26 } & \multicolumn{3}{|c|}{ CX43 } \\
\hline & $\mathrm{n}$ & Positive rate $(\%)$ & P-value & $\mathrm{n}$ & Positive rate $(\%)$ & P-value \\
\hline Paracancerous & 60 & $56 / 60(93.33)$ & $<0.001$ & 60 & $59 / 60(98.34)$ & $<0.001$ \\
\hline Cancer & 60 & $21 / 60(35.00)$ & & 60 & $28 / 60(46.67)$ & \\
\hline
\end{tabular}

Data were compared with Fisher's exact test. CX, connexin.

A

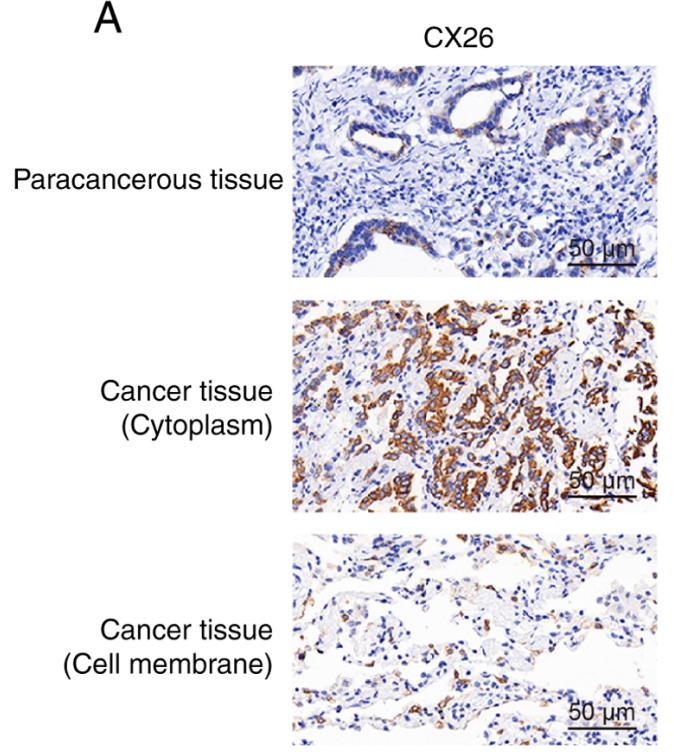

B
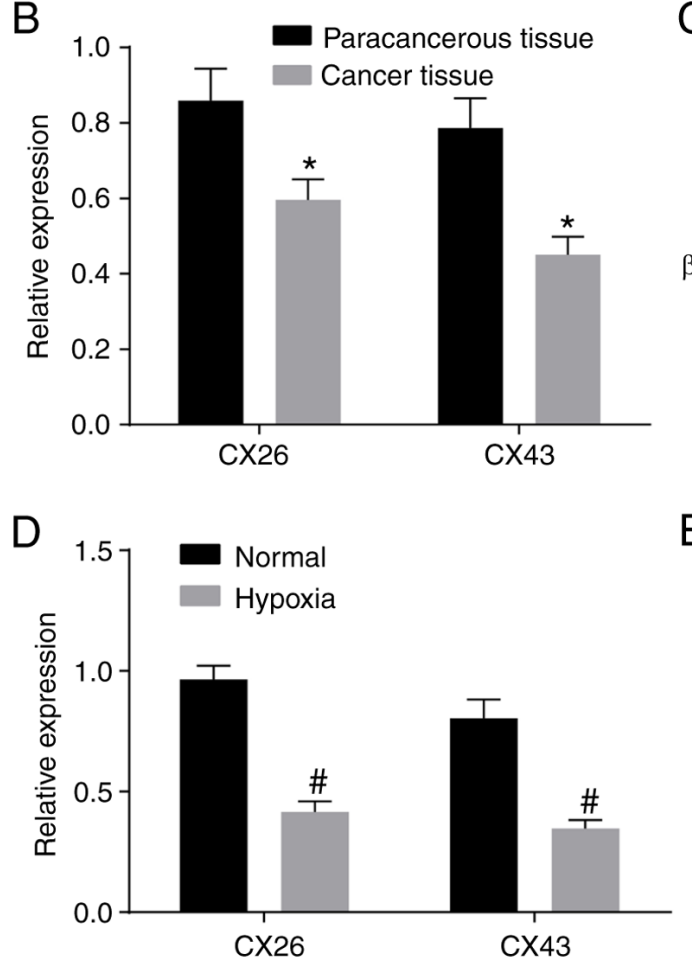

CX43

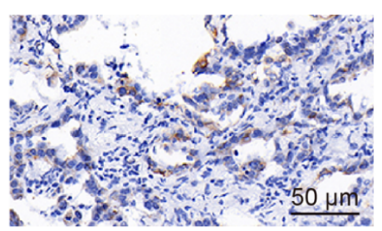

(n)
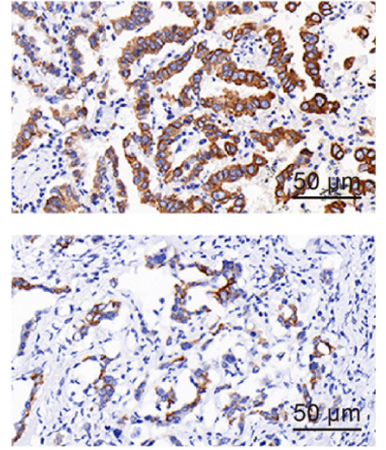

C

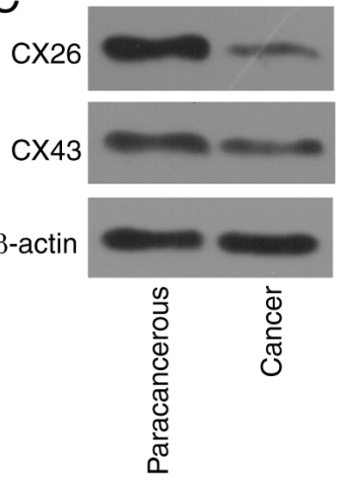

$E$

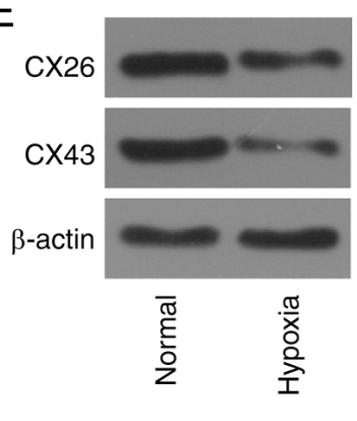

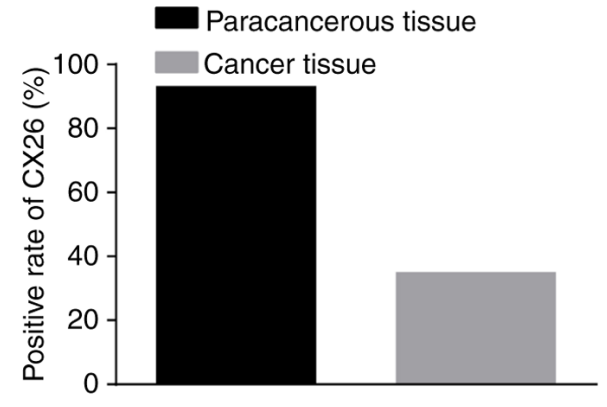

Paracancerous tissue

Cancer tissue
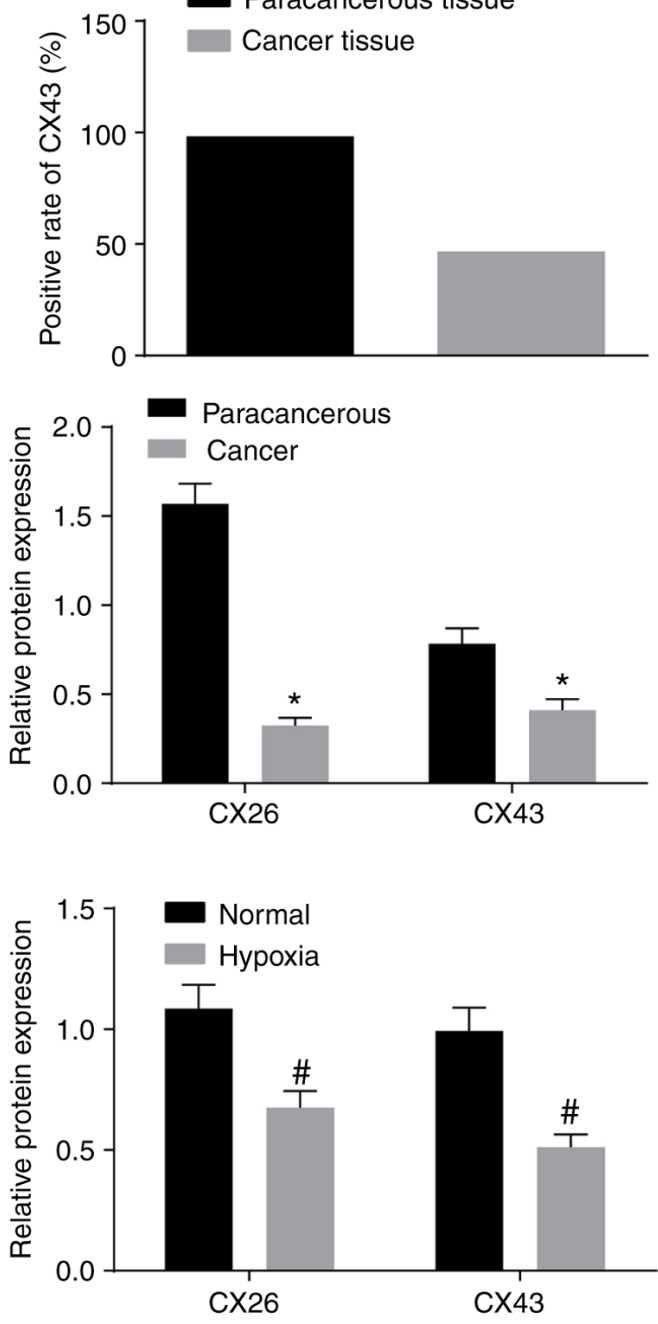

Figure 1. Hypoxia downregulates CX26 and CX43 in PECs. (A) Positive expression of CX26 and CX43 was decreased in cancer tissues as detected by immunohistochemistry (x400; scale bar, $25 \mu \mathrm{m}$; $\mathrm{n}=60$ ). (B) mRNA expression of CX26 and CX43 in pulmonary epithelial tissue as determined by RT-qPCR $(n=60)$. (C) Protein expression of CX26 and CX43 in pulmonary epithelial tissue, as assessed by western blot analysis ( $=60)$. ${ }^{*}<0.05$ vs. paracancerous tissues. (D) mRNA expression of CX26 and CX43 in PECs, as determined by RT-qPCR (n=3). (E) Protein expression of CX26 and CX43 in PECs, as assessed by western blot analysis $(\mathrm{n}=3)$. ${ }^{\text {"}} \mathrm{P}<0.05$ vs. normal group. PECs, pulmonary epithelial cells; RT-qPCR, reverse transcription-quantitative PCR; CX, connexin. 
A
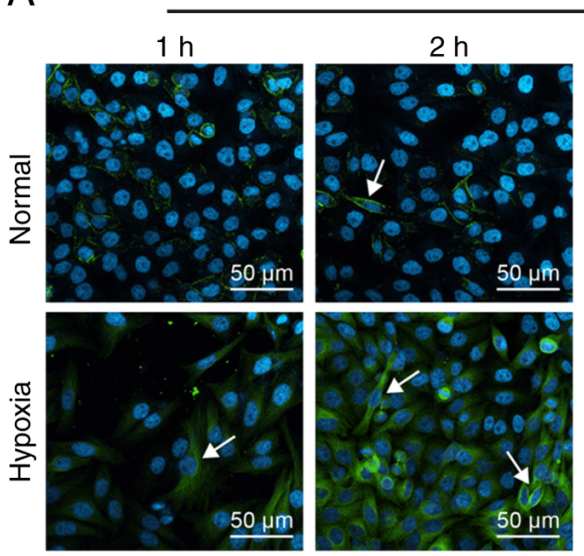

C

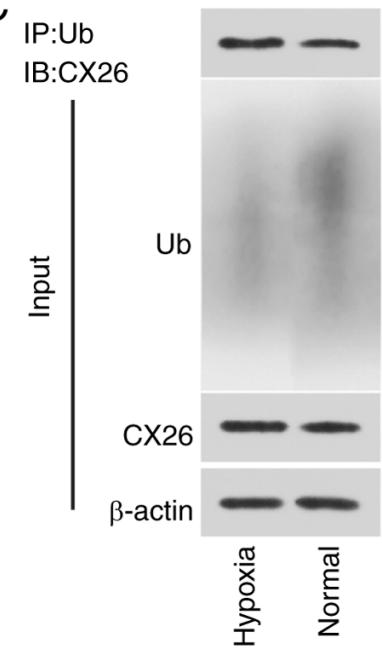

B
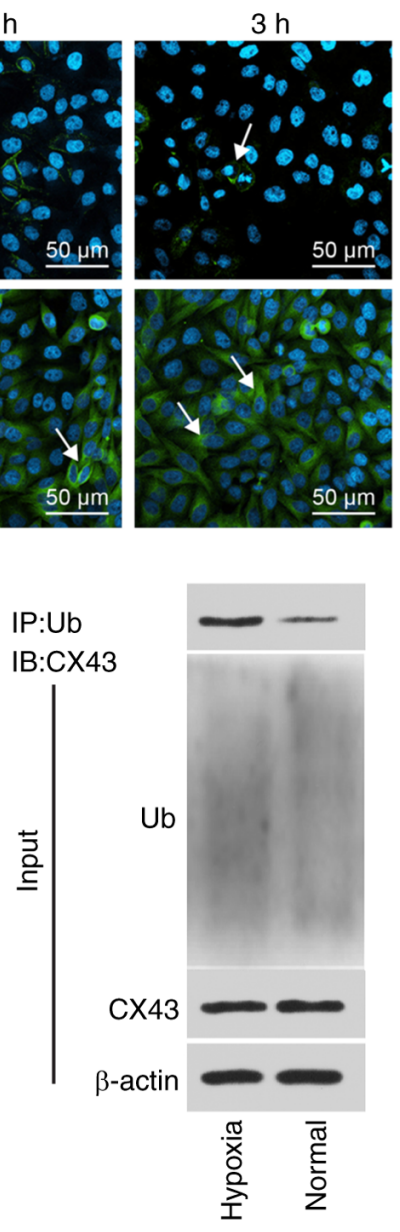

D
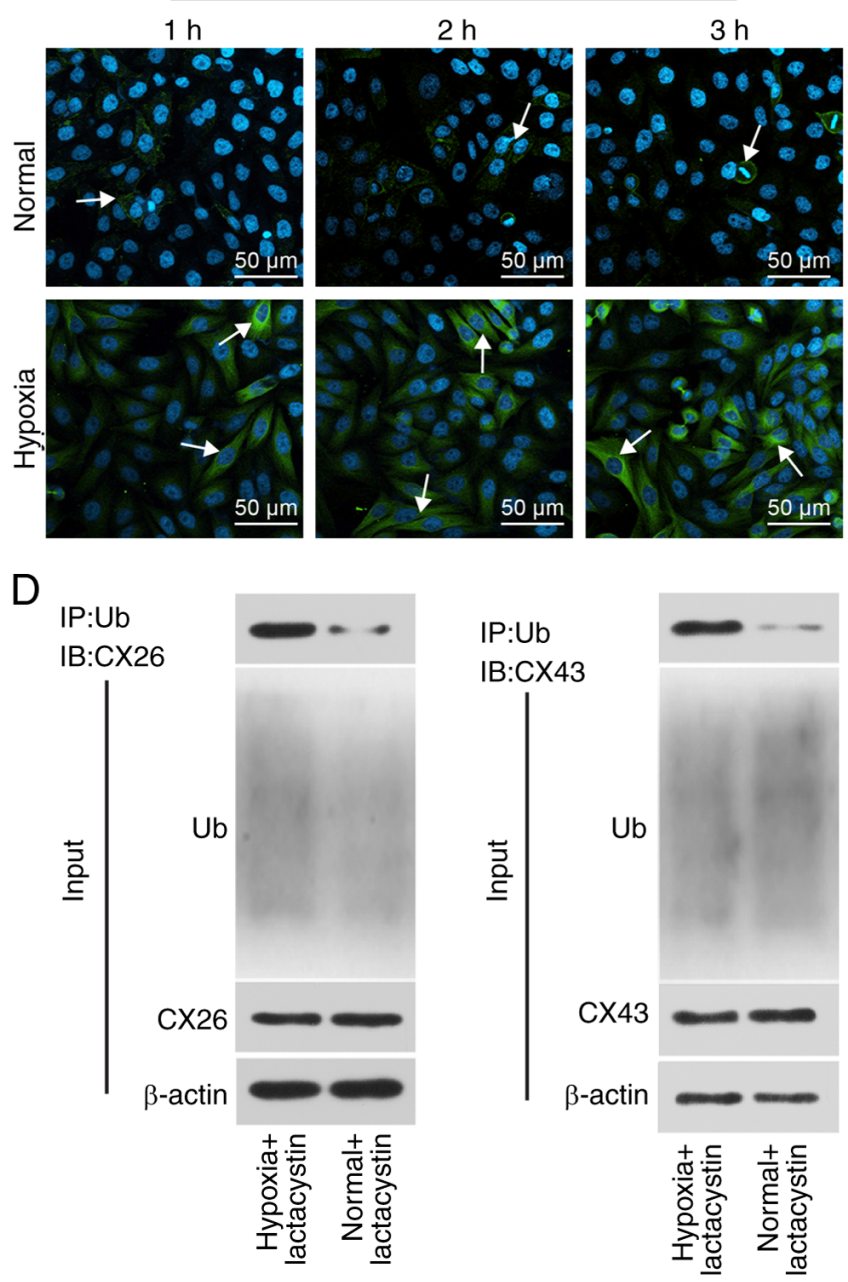

Figure 2. Hypoxia induces internalization and degradation of CX26 and CX43. (A) Localization of CX26, as detected by immunofluorescence staining, where the white arrow indicates CX26 (x200). (B) Localization of CX43, as detected by immunofluorescence staining, where the white arrow indicates CX43 (x200). (C) The binding of CX26 and CX43 to ubiquitin in normal and hypoxia-treated PECs according to Co-IP. (D) The binding of CX26 and CX43 to ubiquitin in normal or hypoxia-treated PECs treated with lactacystin according to Co-IP. PECs, pulmonary epithelial cells; CX, connexin; IP, immunoprecipitation; Ub, ubiquitin; IB, immunoblot.

group, with progression over time (Fig. 2A and B). These findings suggested that expression of CX26 and CX43 in human NSCLC PECs was gradually translocated from the membrane to the cytoplasm following extended periods of hypoxia.

Subsequently, the binding of CX26 and CX43 to ubiquitin was analyzed through a Co-IP, the results of which demonstrated positive signals of CX26 and CX43 in the hypoxia group; however, a weaker signal was detected in the normal group (Fig. 2C). Following long-term hypoxia exposure, PECs were further treated with the proteasome inhibitor lactacystin, after which the binding of CX26 and CX43 to ubiquitin protein was again detected by Co-IP. As illustrated in Fig. 2D, the positive signal of CX26 and CX43 was detected in the hypoxia + lactacystin group, but weaker levels were observed in the normal + lactacystin group. The aforementioned results highlighted that hypoxia promotes the internalization of CX26 and CX43, and ubiquitination and degradation are induced.

Downregulation of CX26 and CX43 induces EMT in NSCLC PECs. To investigate the effects of CX26 and CX43 on EMT in PECs, isolated human NSCLC PECs were exposed to hypoxia. RT-qPCR and western blot analysis were performed to determine the expression of E-cadherin and N-cadherin, while a Transwell assay was performed to assess cell migration ability. The results indicated lower E-cadherin expression and higher $\mathrm{N}$-cadherin expression in the hypoxia group (Fig. 3A-C), while cell migration ability was enhanced (Fig. 3D) compared with the normal group $(\mathrm{P}<0.05)$. Next, human NSCLC PECs were transfected with si-NC-CX26 + si-NC-CX43, si-CX26 + si-NC-CX43, si-NC-CX26+ si-CX43 and si-CX26+ si-CX43. To confirm the interference efficiency of si-CX26 and si-CX43, the expression levels of CX26 and CX43 were determined by RT-qPCR and western blot analysis. It was found that the expression of CX26 in the si-CX26 + si-NC-CX43 and si-CX26 + si-CX43 groups was decreased relative to the si-NC-CX26 + si-NC-CX43 group $(\mathrm{P}<0.05)$, with no significant difference observed in CX26 expression between the si-NC-CX26 + si-CX43 and si-NC-CX26 + si-NC-CX43 groups $(\mathrm{P}>0.05)$. No significant difference was found in $\mathrm{CX} 43$ expression between the si-CX26 + si-NC-CX43 and si-NC-CX26 + si-NC-CX43 

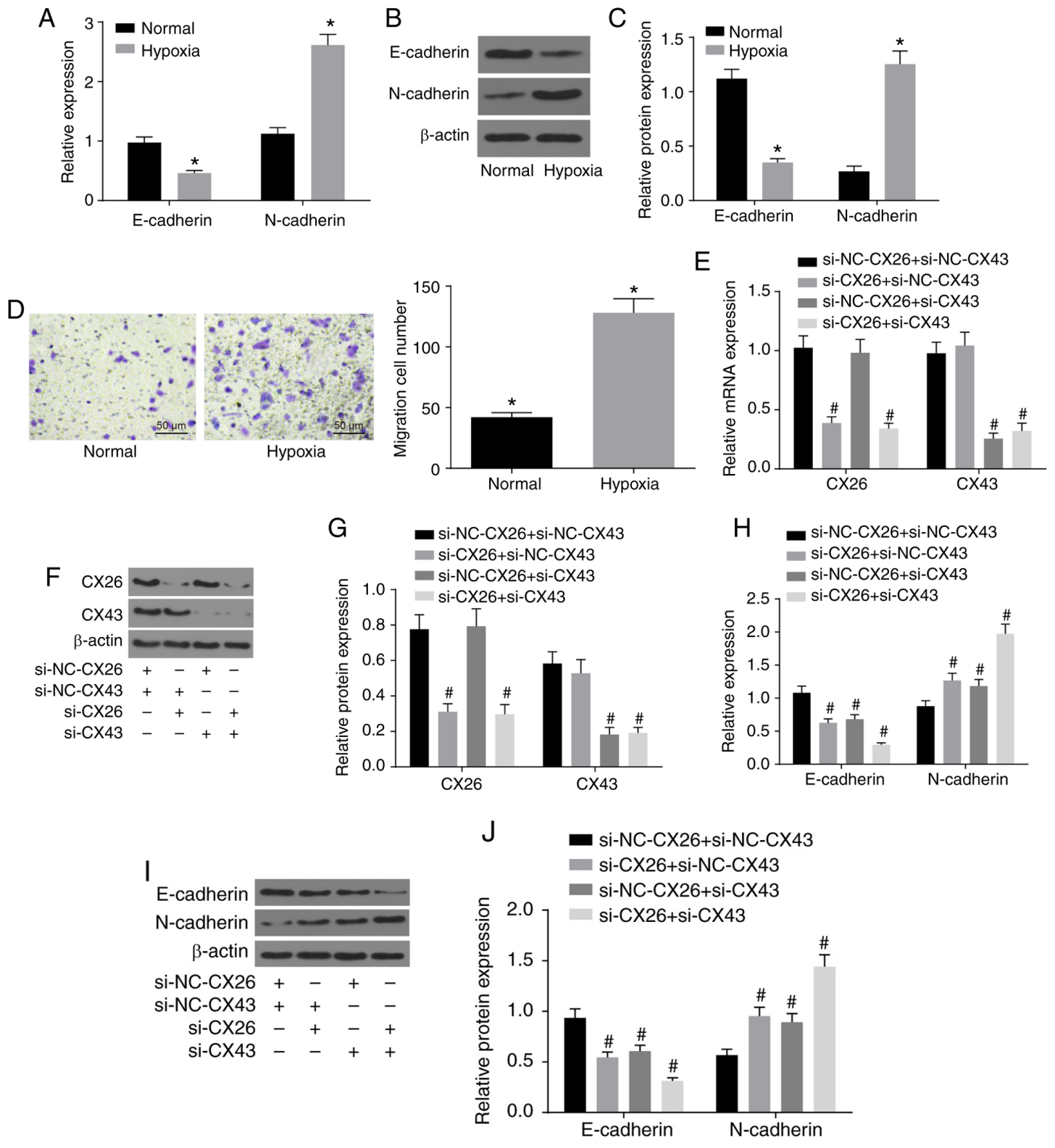
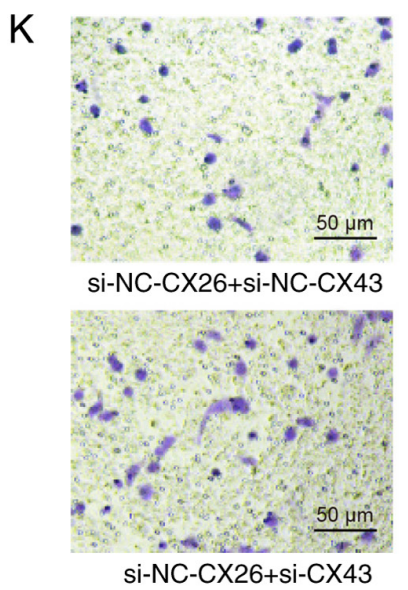

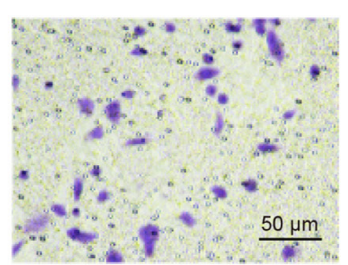
si-CX26+si-NC-CX43

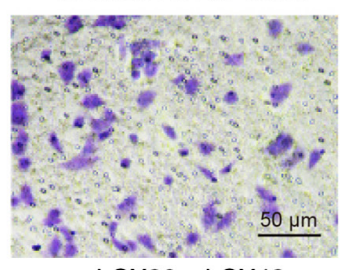

si-CX26+si-CX43

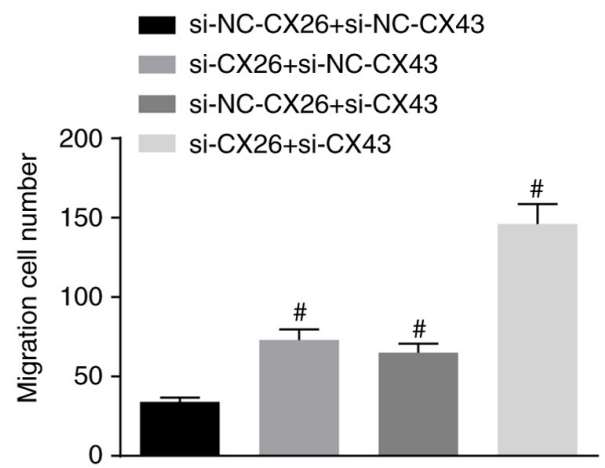

Figure 3. Downregulation of CX26 and CX43 induces epithelial-mesenchymal transition in PECs. (A) mRNA expression of E-cadherin and N-cadherin in human NSCLC PECs following exposure to hypoxia, as evaluated by RT-qPCR. (B) Protein expression of E-cadherin and N-cadherin in human NSCLC PECs following exposure to hypoxia, as assessed by western blot analysis. (C) Semi-quantification of results from panel B. (D) Migration ability of human NSCLC PECs following exposure to hypoxia, as seen by microscopy (x200). Human NSCLC PECs were transfected with si-NC-CX26 + si-NC-CX43, si-CX26 + si-NC-CX43, si-NC-CX26 + si-CX43 or si-CX26 + si-CX43 and (E) mRNA expression of CX26 and CX43 was evaluated by RT-qPCR, (F) protein expression of CX26 and CX43 was assessed by western blot analysis and (G) semi-quantified, (H) mRNA expression of E-cadherin and N-cadherin was evaluated by RT-qPCR, (I) protein expression of E-cadherin and N-cadherin was assessed by western blot analysis and (J) semi-quantified, (K) migration ability was determined (x200). 

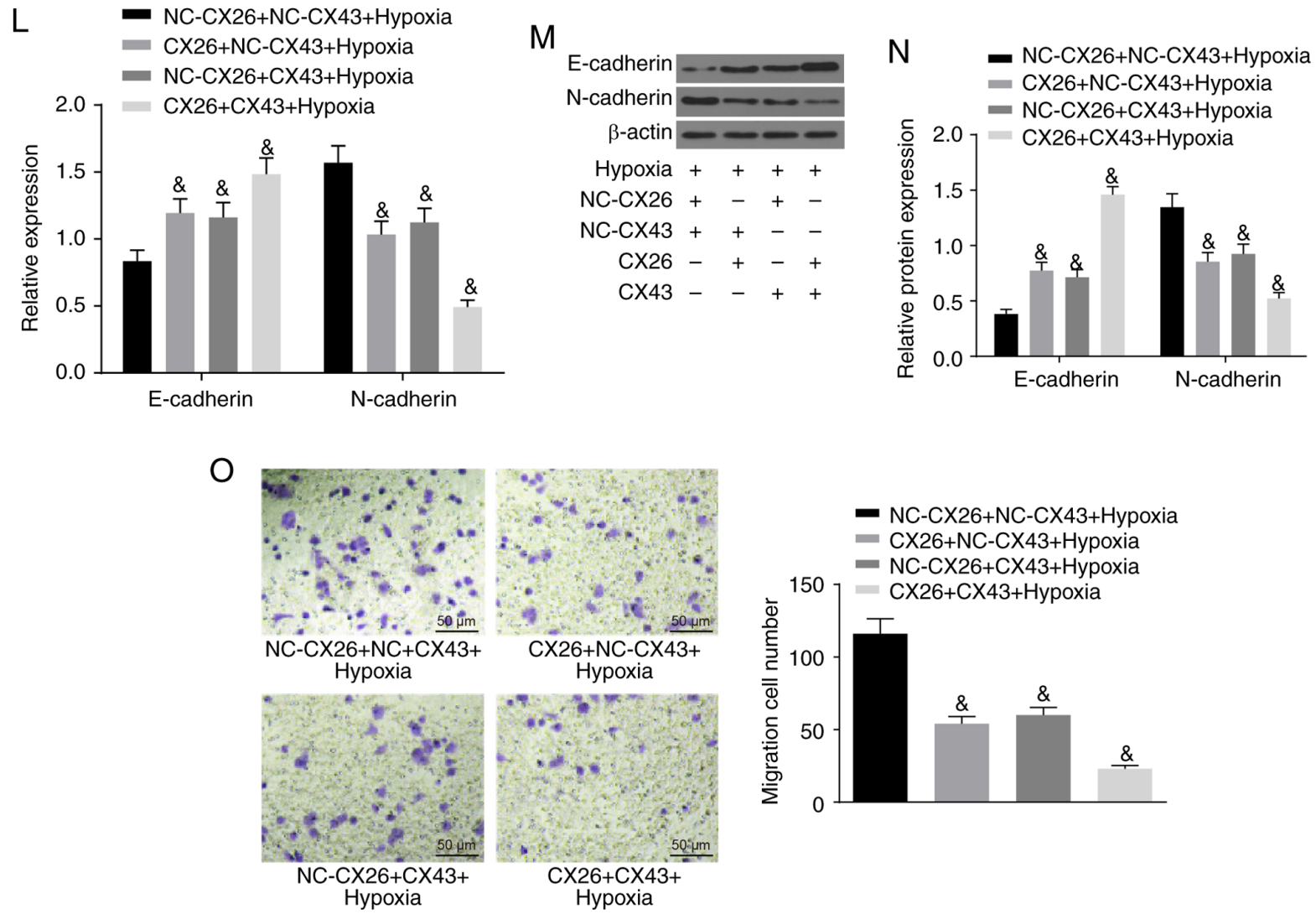

Figure 3. Continued. Downregulation of CX26 and CX43 induces epithelial-mesenchymal transition in PECs. Human NSCLC PECs were transfected with NC-CX26 + NC-CX43, CX26 + NC-CX43, NC-CX26 + CX43 or CX26 + CX43 overexpression vectors, and exposed to hypoxia, then (L) mRNA expression of E-cadherin and N-cadherin was evaluated by RT-qPCR, (M) protein expression of E-cadherin and N-cadherin was assessed by western blot analysis and $(\mathrm{N})$ semi-quantified, and $(\mathrm{O})$ migration ability was determined (x200). ${ }^{*} \mathrm{P}<0.05$ vs. normal group; ${ }^{*} \mathrm{P}<0.05$ vs. si-NC + si-NC group; ${ }^{\&} \mathrm{P}<0.05$ vs. the NC-CX26 + NC-CX43 + hypoxia group (n=3). NSCLC, non-small cell lung cancer; PECs, pulmonary epithelial cells; RT-qPCR, reverse transcription-quantitative PCR; si, small interfering RNA; NC, negative control; CX, connexin.

groups ( $\mathrm{P}>0.05)$, but the expression of CX43 was significantly diminished in the si-CX26+si-CX43 group compared with the si-NC-CX26 + si-NC-CX43 group $(\mathrm{P}<0.05$; Fig. 3E-G). RT-qPCR, western blot analysis and Transwell assay were performed again under hypoxic conditions with $\mathrm{E}$-cadherin and $\mathrm{N}$-cadherin analyzed. The results revealed that, compared with the si-NC-CX26 + si-NC-CX43 group, the si-CX26+si-NC-CX43, si-NC-CX26+si-CX43 and si-CX26 + si-CX43 groups all exhibited decreased E-cadherin expression and increased $\mathrm{N}$-cadherin expression (Fig. $3 \mathrm{H}-\mathrm{J}$ ) along with increased cell migration (Fig. 3K; $\mathrm{P}<0.05$ ). Next, NC-CX26 + NC-CX43, CX26 + NC-CX43, NC-CX26 + CX43 and CX26 + CX43 were used to transfect human NSCLC PECs and overexpress CX26 and CX43, which were then exposed to hypoxic conditions. RT-qPCR, western blot analysis and a Transwell assay were performed for investigative purposes. Compared with the NC-CX26 + NC-CX43 + hypoxia group, elevatedE-cadherin expression, reduced $\mathrm{N}$-cadherin expression (Fig. 3L-N) as well as suppressed cell migration ability (Fig. 3O) were exhibited in the CX26 + NC-CX43 + hypoxia, NC-CX26 + CX43 + hypoxia and CX26 + CX43 + hypoxia groups $(\mathrm{P}<0.05)$. However, no significant difference was observed between the CX26 + NC-CX43 + hypoxia and NC-CX26 + CX43 + hypoxia groups. Based on these results, it was concluded that hypoxia induced EMT in PECs, potentially through the downregulation of CX26 and CX43.
Downregulation of $C X 26$ and $C X 43$ promotes $P E C$ proliferation and growth in NSCLC. Subsequently, to determine the effects of CX26 and CX43 on the proliferation and growth of PECs, human NSCLC PECs were transfected with si-CX26 and/or si-CX43, and an EdU assay was applied to determine cell proliferation. Compared with the si-NC-CX26 + si-NC-CX43 group, cell proliferation ability was promoted in the si-CX26+si-NC-CX43, si-NC-CX26 + si-CX43 and si-CX26 + si-CX43 groups (Fig. 4A). Western blot analysis was performed to determine the relative protein expression of the characteristic stem cell markers Oct4 and Nanog, both of which were elevated in the si-CX26 + si-NC-CX43, si-NC-CX26 + si-CX43 and si-CX26+si-CX43 groups compared with the si-NC-CX26 + si-NC-CX43 group (P<0.05; Fig. 4B). Flow cytometry revealed that the cell apoptosis was decreased in the si-CX26 + si-NC-CX43, si-NC-CX26+si-CX43 and $\mathrm{si-CX} 26+\mathrm{si}-\mathrm{CX} 43$ groups relative to the si-NC-CX26 + si-NC-CX43 group (Fig. 4C) and that cells had been arrested in $\mathrm{S}$ phase (Fig. 4D). As illustrated in Fig. 4E, the colony formation assay revealed that the number of colonies formed was higher in the si-CX26+si-NC-CX43, si-NC-CX26 + si-CX43 and si-CX26 + si-CX43 groups compared with the si-NC-CX26 + si-NC-CX43 group. These findings suggested that CX26 and CX43 were involved in the PEC cell cycle in NSCLC. 

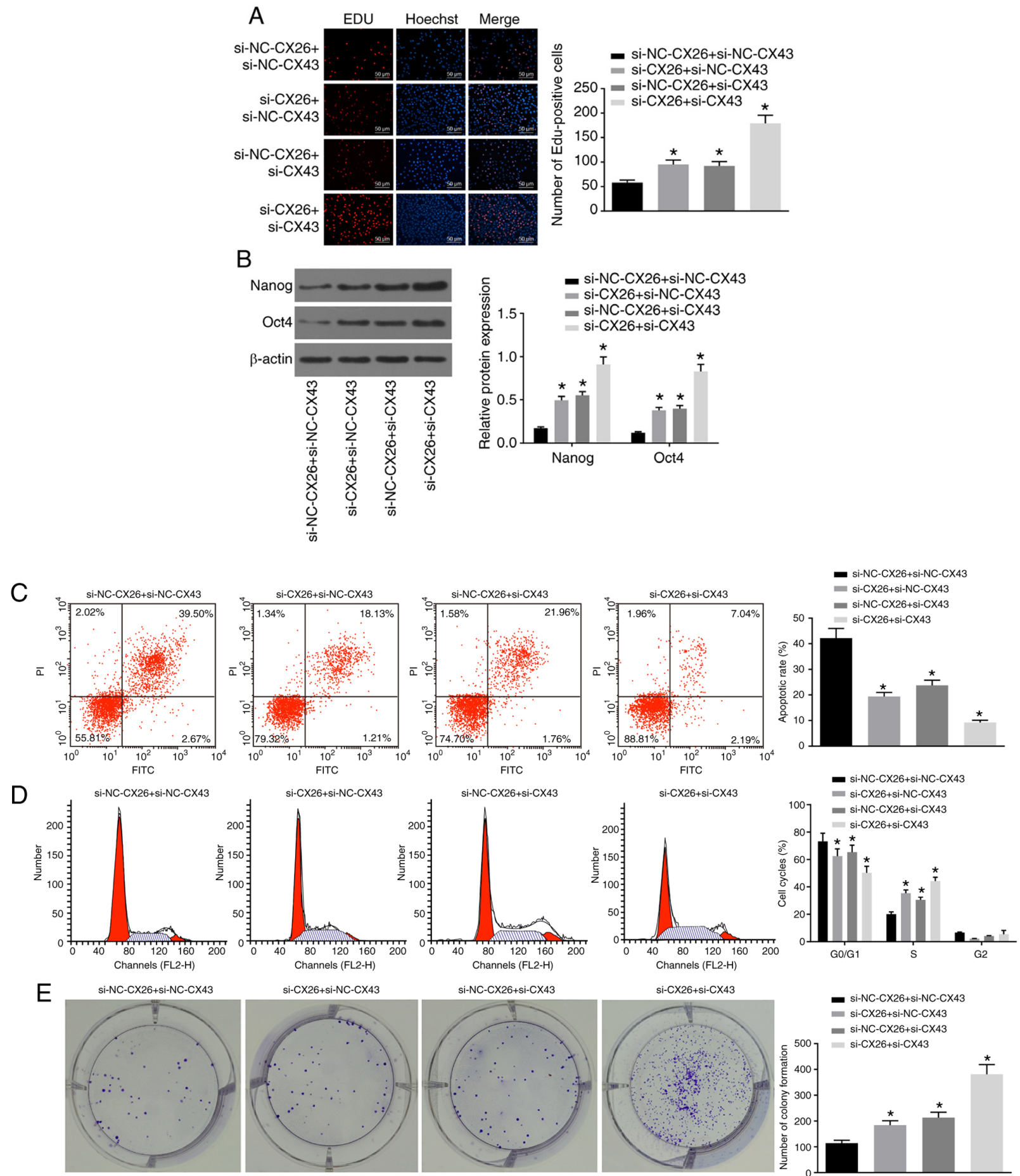

Figure 4. Downregulation of CX26 and CX43 promotes PEC proliferation and growth. PECs were transfected with si-NC-CX26 + si-NC-CX43, si-CX26 + si-NC-CX43, si-NC-CX26 + si-CX43 or si-CX26 + si-CX43. (A) Cell proliferation was assessed by EdU assay (scale bar, $50 \mu \mathrm{m}$ ). (B) Protein expression of Nanog and Oct4 following transfection, as determined by western blot analysis. (C) Cell apoptosis following transfection, as detected by Annexin V-FITC/PI double staining. (D) Cell cycle analysis following transfection, as detected by PI staining. (E) Colony formation in vitro following transfection. PECs were transfected with NC-CX26 + NC-CX43, CX26 + NC-CX43, NC-CX26 + CX43 or CX26 + CX43 and exposed to hypoxia.

Following transfection with CX26 and/or CX43 overexpression vectors, human NSCLC PECs were exposed to hypoxia after which an EdU assay, western blot analysis and flow cytometry were performed to explore the effects on cell function. The results showed that the CX26+ NC-CX43 + hypoxia, NC-CX26 + CX43 + hypoxia and CX26 + CX43 + hypoxia groups had suppressed cell proliferation ability, lower expression of Nanog and Oct4, enhanced cell apoptosis ability and fewer cells in phase $\mathrm{S}$ compared with the NC-CX26 + NC-CX43 + hypoxia group (P<0.05; Fig. 4F-I). However, there were no significant differences detected in relation to the aforementioned changes between the CX26 + NC-CX43 + hypoxia and NC-CX26 + CX43 + hypoxia groups. Taken together, it was concluded that downregulation of CX26 and CX43 promotes PEC proliferation and growth, while inhibiting cell apoptosis in NSCLC cells. 

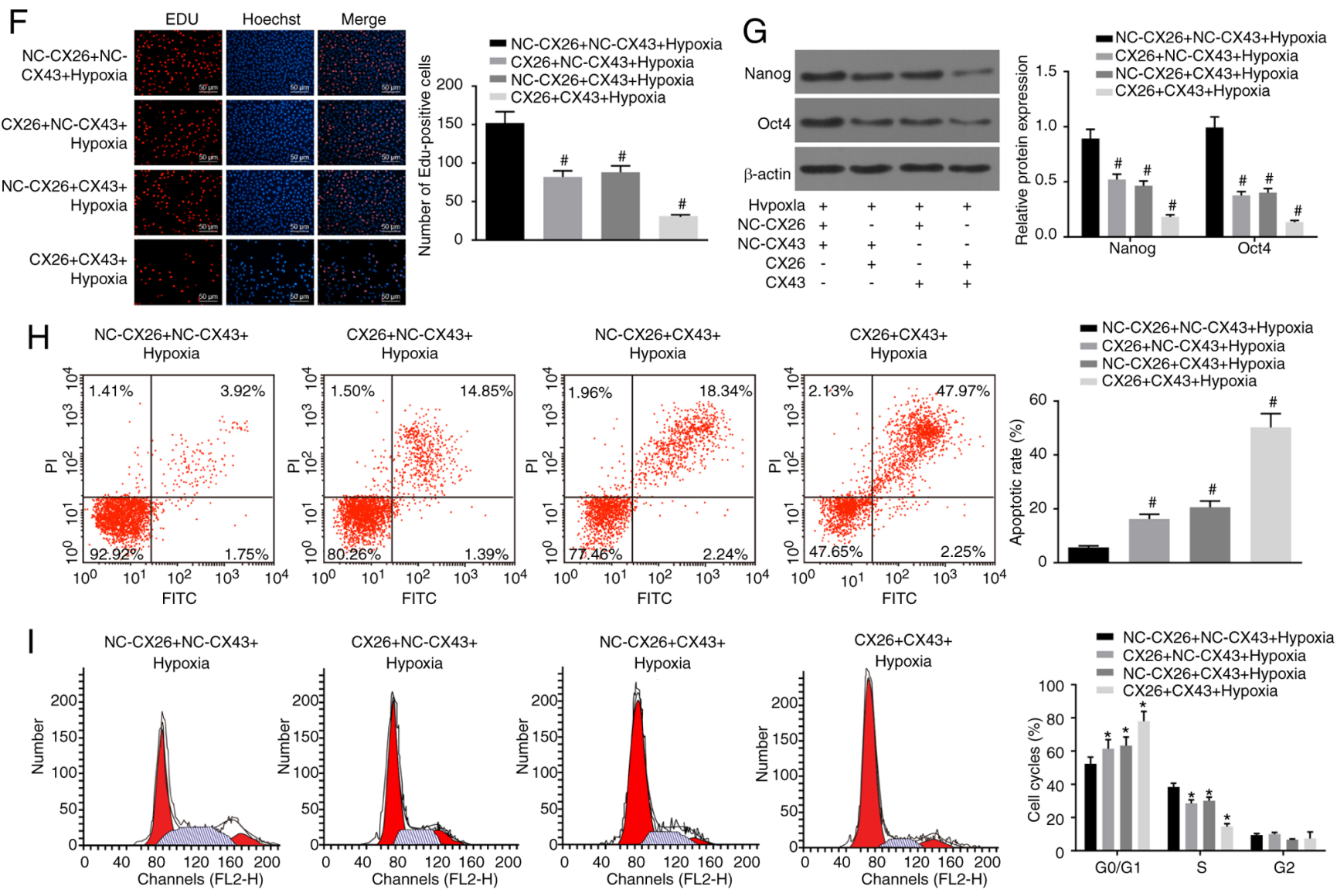

Figure 4. Continued. Downregulation of CX26 and CX43 promotes PEC proliferation and growth. (F) Cell proliferation following transfection was assessed by EdU assay (x200). (G) Protein expression of Nanog and Oct4 following transfection and hypoxia exposure, as determined by western blot analysis. (H) Cell apoptosis following transfection and hypoxia exposure, as detected by Annexin V-FITC/PI double staining. (I) Cell cycle analysis following transfection and hypoxia exposure, as detected by PI single staining. " $\mathrm{P}<0.05$ vs. si-NC-CX26 + si-NC-CX43 group; ${ }^{~} \mathrm{P}<0.05$ vs. NC-CX26 + NC-CX43 + hypoxia group (n=3). PEC, pulmonary epithelial cell; EdU, 5-ethynyl-2'-deoxyuridine; si, small interfering RNA; NC, negative control; CX, connexin; NC, negative control; PI, propidium iodide.

CX26 and CX43 mediate activation of the P53/MDM2 signaling pathway. The association between CX26 and CX43 and their effects on the P53/MDM2 signaling pathway were also investigated based on the quantification of related genes (P53, MDM2 and P21) in human NSCLC PECs using RT-qPCR and western blot analysis. As shown in Fig. 5A-C, the expression of P53 and P21 decreased, while MDM2 expression increased in the hypoxia group cells compared with the normal group $(\mathrm{P}<0.05)$, which demonstrated the activation of the P53/MDM2 signaling pathway under hypoxia.

Additionally, si-CX26 and si-CX43 were used to transfect human NSCLC PECs for further exploration. Compared with the si-NC-CX26 + si-NC-CX43 group, the si-CX26 + si-NC-CX43, si-NC-CX26+si-CX43 and si-CX26 + si-CX43 groups displayed lower expression of $\mathrm{P} 53$ and $\mathrm{P} 21$, along with higher MDM2 expression $(\mathrm{P}<0.05$; Fig. 5D-F), which suggested that the P53/MDM2 signaling pathway was activated through downregulation of CX26 and CX43.

Moreover, protein internalization inhibitors were used to treat human NSCLC PECs that had previously been exposed to hypoxic conditions to assess activation of the P53/MDM2 signaling pathway. The results showed that compared with the normal + MDC group, expression of P53 and P21 was enhanced in the hypoxia + MDC group, whereas MDM2 expression was diminished $(\mathrm{P}<0.05$; Fig. 5G-I). These findings collectively led to the conclusion that hypoxia could activate the P53/MDM2 signaling pathway through induction of CX26 and CX43 internalization.

CX26 and CX43 promote tumorigenesis in vivo. To test the effect of CX26 and CX43 on tumorigenesis in vivo, nude mice were injected with PECs with CX26 and CX43 knockdown. As shown, from the 21st day, tumor growth in the si-CX26+ si-NC-CX43, si-NC-CX26 + si-CX43 and si-CX26 + si-CX43 groups exhibited accelerated growth $(\mathrm{P}<0.05$; Fig. 6A and $\mathrm{B})$ and the nude mice presented with larger tumor weights than those in the si-NC-CX26 + si-NC-CX43 group $(\mathrm{P}<0.05$; Fig. $6 \mathrm{C})$. HE staining was performed to observe tumor metastasis in the lung; compared with normal lung tissues, metastatic tumor cells were closely arranged, with deeply stained nuclear, clear nuclear membrane and obvious nucleolus. Moreover, there were a higher number of tumor metastatic nodes in the si-CX26+si-NC-CX43, si-NC-CX26 + si-CX43 and si-CX26 + si-CX43 groups compared with the si-NC-CX26 + si-NC-CX43 group $(\mathrm{P}<0.05$; Fig. 6D). The data indicated that downregulation of CX26 and CX43 could promote tumorigenesis in vivo. 


\section{A}
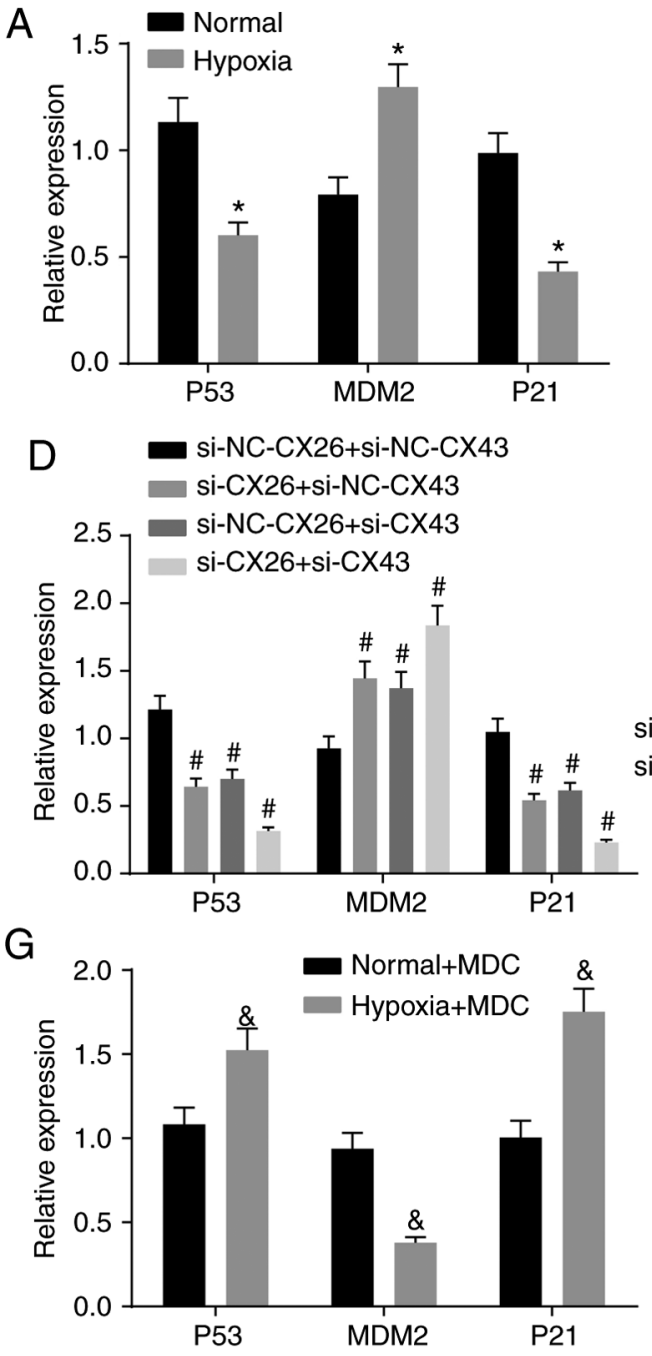

B
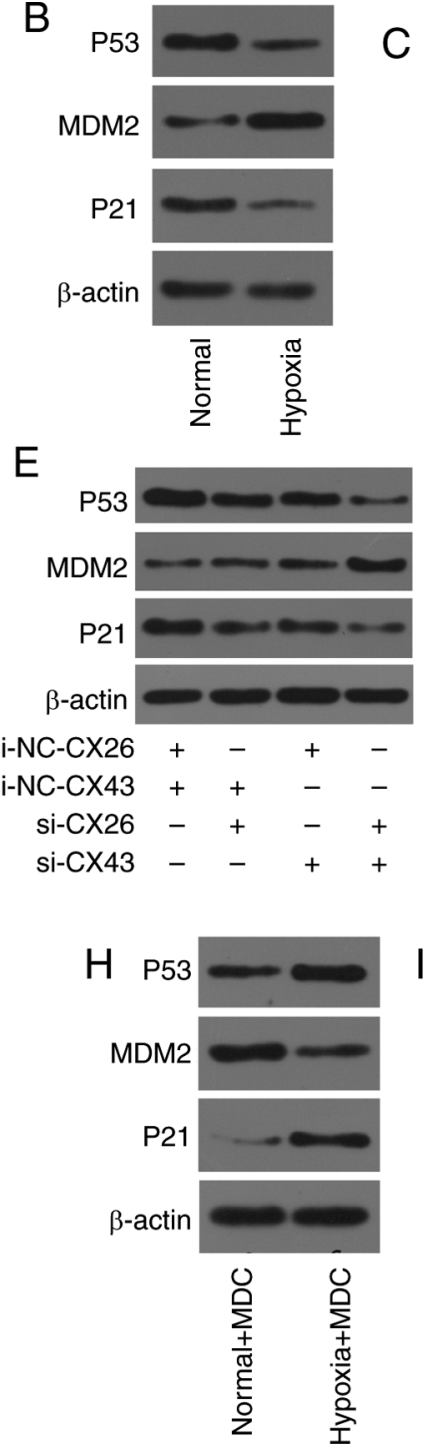

C
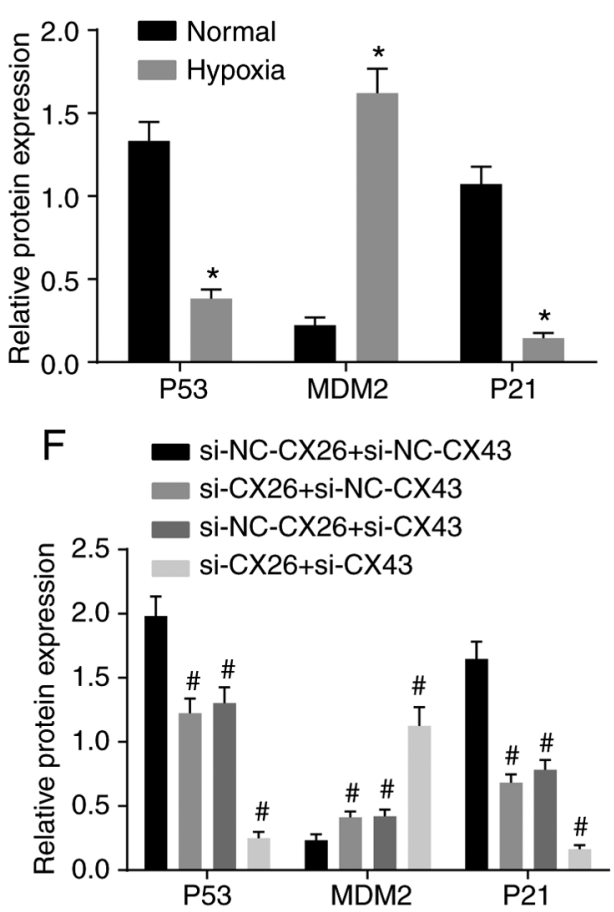

(1in
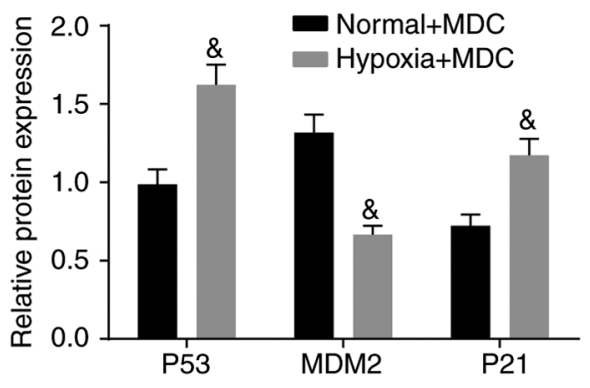

Figure 5. CX26 and CX43 mediate activation of the P53/MDM2 signaling pathway. (A) mRNA and (B) protein expression of P53, MDM2 and P21 in human NSCLC PECs following exposure to hypoxia, as determined by RT-qPCR and western blot analysis, respectively. (C) Semi-quantification of panel B. (D) mRNA and (E) protein expression of P53, MDM2 and P21 in human NSCLC PECs following transfection with si-NC-CX26 + si-NC-CX43, si-CX26 + si-NC-CX43, si-NC-CX26 + si-CX43 or si-CX26 + si-CX43, as tested by RT-qPCR and western blot analysis, respectively. (F) Semi-quantification of panel E. (G) mRNA expression and $(\mathrm{H})$ protein expression of P53, MDM2 and P21 in human NSCLC PECs following MDC treatment and hypoxia exposure, as evaluated by RT-qPCR and western blot analysis, respectively. (I) Semi-quantification of panel H. ${ }^{*} \mathrm{P}<0.05$ vs. normal group; ${ }^{\mathrm{P}}<0.05$ vs. si-NC-CX26 + si-NC-CX43 group; ${ }^{\&} \mathrm{P}<0.05$ vs. the normal + MDC group (n=3). NSCLC, non-small cell cancer; PECs, pulmonary epithelial cells; RT-qPCR, reverse transcription-quantitative PCR; MDM2, murine double minute-2; si, small interfering RNA; NC, negative control; CX, connexin; MDC, monodansylcadaverine.

\section{Discussion}

Lung cancer is widely considered to be among the most prevalent malignancies worldwide, as studies have presented cases of lung cancer in China that account for $>1 / 3$ of all global cases, which places a severe burden on both individuals and society as a whole (23). NSCLC is one of the predominant histological types of lung cancer and includes adenocarcinoma, squamous cell carcinoma and large cell carcinoma, all of which usually arise from the alveoli, small bronchi or bronchioles (24). The alveoli, the structures where gas transfer occurs, play a central role in lung function. Hypoxia causes lung dysfunction as a result of impaired tissue function (25). Mounting evidence has continued to highlight the significance of CX isotypes in human cells partially due to their ubiquity and large density in solid tissues (26). In the current study, we investigated the roles of CX26 and CX43 under hypoxic conditions in the progression of NSCLC through a series of well-designed experiments and subsequently concluded that hypoxia could induce the internalization of CX26 and CX43 via the P53/MDM2 signaling pathway, which ultimately further promotes the incidence of NSCLC.

Initially, a key finding of our study indicated aberrant expression of CX26 and CX43 in PECs induced by hypoxia, which suggests that CX26 and CX43 were both downregulated in cancer tissues, as well as cancer cells, after hypoxia exposure. Likewise, researchers have reported that CX43 expression is reduced in NSCLC, while demonstrating that the downregulation of CX26/CX43 is also found in the alveolar 

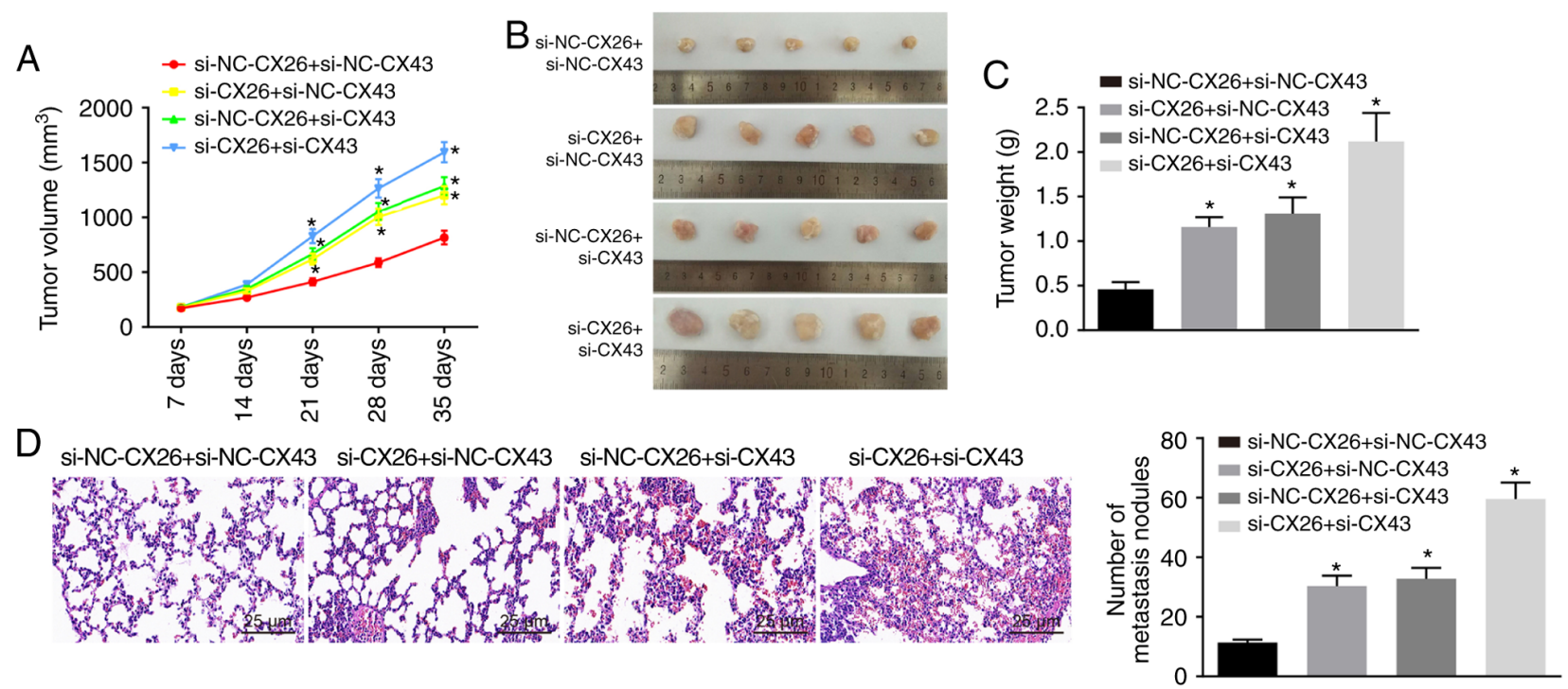

Figure 6. Downregulation of CX26 and CX43 promotes tumorigenesis in vivo. (A) Tumor volume, (B) images of tumors in nude mice, (C) tumor weight and (D) hematoxylin-eosin staining (x200) of lung metastases in nude mice in the si-NC-CX26 + si-NC-CX43, si-CX26 + si-NC-CX43, si-NC-CX26 + si-CX43 or si-CX26 + si-CX43 groups. "P<0.05 vs. si-NC-CX26 + si-NC-CX43 group. si, small interfering RNA; NC, negative control; CX, connexin; NC, negative control.

epithelium in human lung cancer (27). Such changes have been widely discussed in previous studies, which have indicated that CX overexpression is observed at the early stage of cancer, while reductions in function and expression have been noted in malignant tumors, and at times, expression is even absent at more advanced stages (26). Similar results have been presented in another study in which subsequent changes, including lung injury, induced the downregulation of CX43 and reduced gap junctional intercellular communication (28). Furthermore, the role ofCX-involved channels has been shown to contribute to lung repair and inflammatory response (27). It has been confirmed by previous studies that hypoxia reduces the expression of CX26 and CX43. Specifically, chronic prenatal hypoxia causes promoter region hypermethylation and decreases the mRNA and protein expression of CX26 (29). Additionally, hypoxia is observed to decrease the CX43 protein level by $30-50 \%$ (30), which was consistent with the results of the present study.

Furthermore, additional evidence has been presented in the current study that demonstrates that hypoxic conditions promote the binding of CX26 and CX43 to ubiquitin protein in favor of CX26 and CX43 internalization and degradation. In addition, the expression CX26/CX43 was predominantly located within the cytoplasm as opposed to the membrane. A prior study revealed that following extended exposure to hypoxia, CX43 may translocate to the cytoplasm from the membrane, which is accompanied by a decrease in communication properties (31); this was largely consistent with the results of the current study. Ubiquitination is a crucial posttranslational modification occurring during nonlysosomal protein degradation that is capable of influencing the progression and development of cancer; ubiquitination also shares a relationship with both tumor suppressive and stimulatory pathways (32). Additionally, other studies have previously indicated that degradation of CX co-exists with a variety of physiological and pathological variations, including cell migration (33), which was further verified by the results of the present study.
In a subsequent experiment, the effects involved the regulation of the biological functions of NSCLC PECs EMT and growth involving CX26 and CX43 were determined to be enhanced when CX26 and CX43 were downregulated, which was further evidenced by the increased expression of N-cadherin, Nanog and Oct4, decreased E-cadherin expression and activation of the P53/MDM2 signaling pathway. A conclusion drawn from the experiment performed by Zhao et al (34) suggested that, among the Chinese population, expression of CX43 and E-cadherin decreases during NSCLC development, and thus the differentiation and lymph node metastasis of cancer cells may be predicted. Oct4 and its downstream target Nanog have been investigated in depth as essential homeobox transcription factors that participate in stem cell self-renewal fate determination, while the ectopic overexpression of both has been observed to operate in tandem to promote EMT and tumor-initiating ability of lung adenocarcinoma, the most prevalent histologic type of lung cancer (35). Evidence has been previously presented that indicates that, if activated under hypoxic conditions, P53 is able to function as an extraordinary tumor suppressor regarding progression and prognosis through its negative interaction with MDM2 (36). The function of P53 is largely linked to cell proliferation and apoptosis, along with cell cycle distribution, as P53 can promote cell apoptosis (37). The follow-up in vivo experiments further verified that downregulated CX26 and CX43 promoted tumorigenicity. The aforementioned literature and the observations of this study support the promotive role of CX26 and CX43 in NSCLC.

Taken together, the data obtained in the current study confirmed that hypoxia reduced the expression of CX26 and CX43, and abnormal activation of the P53/MDM2 signaling pathway through a combination of effects. These effects regulated proliferation, migration and apoptosis of PECs, which ultimately promoted NSCLC progression. Thus, 
in accordance with the evidence presented, it is proposed that, under hypoxic conditions, CX26 and CX43 may have therapeutic implications. Nevertheless, this is a relatively new field of research in pulmonary diseases and provides a platform for a better understanding of the mechanisms of NSCLC progression. Therefore, more studies and additional research areas are required to establish enhanced treatment methods for patients with NSCLC. In the follow-up experiments, in addition to RT-qPCR and western blot analysis performed to detect the efficiency of RNAi, gene overexpression experiments for gene interference will be conducted to ensure the specificity of RNAi.

\section{Acknowledgements}

Not applicable.

\section{Funding}

No funding was received.

\section{Availability of data and materials}

The datasets used and/or analyzed during the current study are available from the corresponding author on reasonable request.

\section{Authors' contributions}

JZ and SZ performed and analyzed the studies. SZ and XL initiated the work and analyzed the data. XL and JCL obtained the results and validated them, and provided the figures and tables and wrote the manuscript. All authors contributed to the revised manuscript and approved the final manuscript.

\section{Ethics approval and consent to participate}

The study was approved by the Ethics Committee of The First Affiliated Hospital of Nanchang University. Written informed consent was obtained from each participant. All animal experimentation in this study was approved by the Institutional Animal Care and Use Committee of The First Affiliated Hospital of Nanchang University.

\section{Patient consent for publication}

Not applicable.

\section{Competing interests}

The authors declare that they have no competing interests.

\section{References}

1. Siegel RL, Miller KD and Jemal A: Cancer statistics, 2019. CA Cancer J Clin 69: 7-34, 2019.

2. Wakelee H, Kelly $\mathrm{K}$ and Edelman MJ: 50 Years of progress in the systemic therapy of non-small cell lung cancer. Am Soc Clin Oncol Educ Book: 177-189, 2014.

3. Lei Z, Xu G, Wang L, Yang H, Liu X, Zhao J and Zhang HT: MiR-142-3p represses TGF- $\beta$-induced growth inhibition through repression of TGFbetaR1 in non-small cell lung cancer. FASEB J 28: 2696-2704, 2014.
4. Grutsch JF, Wood PA, Du-Quiton J, Reynolds JL, Lis CG Levin RD, Ann Daehler M, Gupta D, Quiton DF and Hrushesky WJ: Validation of actigraphy to assess circadian organization and sleep quality in patients with advanced lung cancer. J Circadian Rhythms 9: 4, 2011.

5. Kleisiaris CF, Kritsotakis EI, Daniil Z, Tzanakis N, Papaioannou A and Gourgoulianis KI: The prevalence of obstructive sleep apnea-hypopnea syndrome-related symptoms and their relation to airflow limitation in an elderly population receiving home care. Int J Chron Obstruct Pulmon Dis 9: 1111-1117, 2014.

6. Bouloukaki I, Papadimitriou V, Sofras F, Mermigkis C, Moniaki V, Siafakas NM and Schiza SE: Abnormal cytokine profile in patients with obstructive sleep apnea-hypopnea syndrome and erectile dysfunction. Mediators Inflamm 2014: 568951, 2014

7. Yang J, Qin G, Luo M, Chen J, Zhang Q, Li L, Pan L and Qin S: Reciprocal positive regulation between Cx26 and PI3K/Akt pathway confers acquired gefitinib resistance in NSCLC cells via GJIC-independent induction of EMT. Cell Death Dis 6: e1829, 2015.

8. Crespin S, Bacchetta M, Bou Saab J, Tantilipikorn P, Bellec J, Dudez T, Nguyen TH, Kwak BR, Lacroix JS, Huang S, et al: $\mathrm{Cx} 26$ regulates proliferation of repairing basal airway epithelial cells. Int J Biochem Cell Biol 52: 152-160, 2014.

9. Garcia IE, Maripillán J, Jara O, Ceriani R, Palacios-Munoz A, Ramachandran J, Olivero P, Perez-Acle T, González C, Sáez JC, et al: Keratitis-ichthyosis-deafness syndrome-associated Cx26 mutants produce nonfunctional gap junctions but hyperactive hemichannels when co-expressed with wild type Cx43. J Invest Dermatol 135: 1338-1347, 2015.

10. Sulkowska U, Febp AW and Sulkowski S: Association of STAT3 with $\mathrm{Cx} 26$ and $\mathrm{Cx} 43$ in human uterine endometrioid adenocarcinoma. Oncol Lett 11: 4134-4138, 2016.

11. Deben C, Wouters A, Op de Beeck K, van Den Bossche J, Jacobs J, Zwaenepoel K, Peeters M, Van Meerbeeck J, Lardon F, Rolfo C, et al: The MDM2-inhibitor Nutlin-3 synergizes with cisplatin to induce p53 dependent tumor cell apoptosis in non-small cell lung cancer. Oncotarget 6: 22666-22679, 2015.

12. Chang H, Li C, Huo K, Wang Q, Lu L, Zhang Q, Wang Y and Wang W: Luteolin prevents $\mathrm{H} 2 \mathrm{O} 2$-induced apoptosis in $\mathrm{H} 9 \mathrm{C} 2$ cells through modulating Akt-P53/Mdm2 signaling pathway. Biomed Res Int 2016: 5125836, 2016.

13. Reddy R, Buckley S, Doerken M, Barsky L, Weinberg K, Anderson KD, Warburton D and Driscoll B: Isolation of a putative progenitor subpopulation of alveolar epithelial type 2 cells. Am J Physiol Lung Cell Mol Physiol 286: L658-L667, 2004.

14. Bao B, Groves K, Zhang J, Handy E, Kennedy P, Cuneo G, Supuran CT, Yared W, Rajopadhye M and Peterson JD: In vivo imaging and quantification of carbonic anhydrase IX expression as an endogenous biomarker of tumor hypoxia. PLoS One 7: e50860, 2012.

15. Tang D, Yan T, Zhang J, Jiang X, Zhang D and Huang Y: Notch1 signaling contributes to hypoxia-induced high expression of Integrin $\beta 1$ in keratinocyte migration. Sci Rep 7: 43926, 2017.

16. Hau S, Reich DM, Scholz M, Naumann W, Emmrich F, Kamprad M and Boltze J: Evidence for neuroprotective properties of human umbilical cord blood cells after neuronal hypoxia in vitro. BMC Neurosci 9: 30, 2008.

17. Bouley R, Lin HY, Raychowdhury MK, Marshansky V, Brown D and Ausiello DA: Downregulation of the vasopressin type 2 receptor after vasopressin-induced internalization: Involvement of a lysosomal degradation pathway. Am J Physiol Cell Physiol 288: C1390-C1401, 2005.

18. Kim SJ, Kim MY, Lee EJ, Ahn YS and Baik JH: Distinct regulation of internalization and mitogen-activated protein kinase activation by two isoforms of the dopamine D2 receptor. Mol Endocrinol 18: 640-652, 2004.

19. Atkins D, Reiffen KA, Tegtmeier CL, Winther H, Bonato MS and Storkel S: Immunohistochemical detection of EGFR in paraffin-embedded tumor tissues: Variation in staining intensity due to choice of fixative and storage time of tissue sections. J Histochem Cytochem 52: 893-901, 2004.

20. Livak KJ and Schmittgen TD: Analysis of relative gene expression data using real-time quantitative PCR and the 2(-Delta Delta C(T)) method. Methods 25: 402-408, 2001.

21. Wilmanns C, Fan D, O'Brian CA, Bucana CD and Fidler IJ: Orthotopic and ectopic organ environments differentially influence the sensitivity of murine colon carcinoma cells to doxorubicin and 5-fluorouracil. Int J Cancer 52: 98-104, 1992. 
22. Schlagbauer-Wadl H, Griffioen M, van Elsas A, Schrier PI, Pustelnik T, Eichler HG, Wolff K, Pehamberger H and Jansen B: Influence of increased c-Myc expression on the growth characteristics of human melanoma. J Invest Dermatol 112: 332-336, 1999.

23. Hong QY, Wu GM, Qian GS, Hu CP, Zhou JY, Chen LA, Li WM, Li SY, Wang K, Wang Q, et al: Prevention and management of lung cancer in China. Cancer 121 (Suppl 17): S3080-S3088, 2015.

24. Long F, Su JH, Liang B, Su LL and Jiang SJ: Identification of gene biomarkers for distinguishing small-cell lung cancer from non-small-cell lung cancer using a network-based approach. Biomed Res Int 2015: 685303, 2015.

25. Olmeda B, Umstead TM, Silveyra P, Pascual A, Lopez-Barneo J, Phelps DS, Floros J and Perez-Gil J: Effect of hypoxia on lung gene expression and proteomic profile: Insights into the pulmonary surfactant response. J Proteomics 101: 179-191, 2014.

26. Teleki I, Szasz AM, Maros ME, Gyorffy B, Kulka J, Meggyeshazi N, Kiszner G, Balla P, Samu A and Krenacs T: Correlations of differentially expressed gap junction connexins Cx26, Cx30, Cx32, Cx43 and Cx46 with breast cancer progression and prognosis. PLoS One 9: e112541, 2014.

27. Losa D, Chanson M and Crespin S: Connexins as therapeutic targets in lung disease. Expert Opin Ther Targets 15: 989-1002, 2011.

28. Liu T, Li Y, Zhang B, Ma L, Liu W, Li Z and Jin F: The role of phosphorylated Cx43 on PKC mediated Ser368 in lung injury induced by seawater inhalation. Inflammation 38: 1847-1854, 2015.

29. Lin J, Huang H, Lv G, Xu X, Lin W, Xu X, Cheng J and Zheng M: Chronic prenatal hypoxia impairs cochlear development, a mechanism involving connexin26 expression and promoter methylation. Int J Mol Med 41: 852-858, 2018.
30. Wu X, Huang W, Luo G and Alain LA: Hypoxia induces connexin 43 dysregulation by modulating matrix metalloproteinases via MAPK signaling. Mol Cell Biochem 384: 155-162, 2013.

31. Otto T, Gellhaus A, Lüschen N, Scheidler J, Bendix I, Dunk C, Wolf N, Lennartz K, Koninger A, Schmidt M, et al: Oxygen sensitivity of placental trophoblast Connexins 43 and 46: A role in preeclampsia? J Cell Biochem 116: 2924-2937, 2015.

32. Morrow JK, Lin HK, Sun SC and Zhang S: Targeting ubiquitination for cancer therapies. Future Med Chem 7: 2333-2350, 2015.

33. Falk MM, Kells RM and Berthoud VM: Degradation of connexins and gap junctions. FEBS Lett 588: 1221-1229, 2014.

34. Zhao JQ, Sun FJ, Liu SS, Yang J, Wu YQ, Li GS, Chen QY and Wang JX: Expression of connexin 43 and E-cadherin protein and mRNA in non-small cell lung cancers in Chinese patients. Asian Pac J Cancer Prev 14: 639-643, 2013.

35. Chiou SH, Wang ML, Chou YT, Chen CJ, Hong CF, Hsieh WJ, Chang HT, Chen YS, Lin TW, Hsu HS and Wu CW: Coexpression of Oct4 and Nanog enhances malignancy in lung adenocarcinoma by inducing cancer stem cell-like properties and epithelial-mesenchymal transdifferentiation. Cancer Res 70: 10433-10444, 2010.

36. Wang W and Hu Y: Small molecule agents targeting the p53-MDM2 pathway for cancer therapy. Med Res Rev 32: 1159-1196, 2012.

37. Zhang P, Kratz AS, Salama M, Elabd S, Heinrich T, Wittbrodt J, Blattner C and Davidson G: Expression screening using a Medaka cDNA library identifies evolutionarily conserved regulators of the $\mathrm{p53/Mdm} 2$ pathway. BMC Biotechnol 15: 92, 2015. International (CC BY-NC-ND 4.0) License. 